\title{
ALMOST FORMALITY OF MANIFOLDS OF LOW DIMENSION
}

\author{
DOMENICO FIORENZA, KOTARO KAWAI, HÔNG VÂN LÊ, \\ AND LORENZ SCHWACHHÖFER
}

\begin{abstract}
In this paper we introduce the notion of Poincaré DGCAs of Hodge type, which is a subclass of Poincaré DGCAs encompassing the de Rham algebras of closed orientable manifolds. Then we introduce the notion of the small algebra and the small quotient algebra of a Poincaré DGCA of Hodge type. Using these concepts, we investigate the equivalence class of $(r-1)$ connected $(r>1)$ Poincaré DGCAs of Hodge type. In particular, we show that a $(r-1)$ connected Poincaré DGCA of Hodge type $\mathcal{A}^{*}$ of dimension $n \leq 5 r-3$ is $A_{\infty^{-}}$ quasi-isomorphic to an $A_{3}$-algebra and prove that the only obstruction to the formality of $\mathcal{A}^{*}$ is a distinguished Harrison cohomology class $\left[\mu_{3}\right] \in \operatorname{Harr}^{3,-1}\left(H^{*}\left(\mathcal{A}^{*}\right), H^{*}\left(\mathcal{A}^{*}\right)\right)$. Moreover, the cohomology class $\left[\mu_{3}\right]$ and the DGCA isomorphism class of $H^{*}\left(\mathcal{A}^{*}\right)$ determine the $A_{\infty}$-quasiisomorphism class of $\mathcal{A}^{*}$. This can be seen as a Harrison cohomology version of the Crowley-Nordström results CN2015 on rational homotopy type of $(r-1)$-connected $(r>1)$ closed manifolds of dimension up to $5 r-3$. We also derive the almost formality of closed $G_{2}$-manifolds, which have been discovered recently by Chan-Karigiannis-Tsang in [CKT2018, from our results and the Cheeger-Gromoll splitting theorem.
\end{abstract}

\section{INTRODUCTION}

By the seminal work of Sullivan Sullivan1977] it is known that the equivalence class of the de Rham algebra of a closed simply connected manifold determines its real homotopy type. Thus, it is desirable to obtain invariants which measure the formality of the de Rham algebra, and more generally identify the rational homotopy type of a manifold, and the present paper aims to describe such invariants under suitable highly enough connectedness assumptions. Assuming (as we shall always do) that the underlying manifold

Date: February 23, 2021.

2010 Mathematics Subject Classification. Primary:57R19, Secondary:53C25, 53C29, $58 \mathrm{~A} 10$

Key words and phrases. Poincaré differential graded algebra, formality, $A_{\infty}$-algebra, Massey product, Cheeger-Gromoll splitting theorem, $G_{2}$-manifold.

The second named author is supported by JSPS KAKENHI Grant Numbers JP17K14181, and the research of the third named author is supported by the GAČproject 18-00496S and RVO:67985840. The fourth named author was supported by the Deutsche Forschungsgemeinschaft by grant SCHW 893/5-1. 
is closed, connected and oriented, the de Rham algebra is a Poincaré differential graded commutative algebra, meaning that there is a top homology class which induces a non-degenerate pairing on the cohomology algebra.

This work is inspired by two recent approaches to this question. The first is the article CKT2018 where it is shown that the de Rham algebra of a $G_{2^{-}}$ manifold (i.e., a closed 7-manifold with a torsion-free $G_{2}$-structure) is almost formal, i.e., equivalent to a DGCA all of whose differentials vanish except in the middle degree. Second, in [CN2015] a new invariant for a Poincaré DGCA is introduced, called the Bianchi-Massey tensor and it is shown that this tensor is the only obstruction to formality of $(r-1)$-connected $(r>1)$ closed manifolds in dimension at most $5 r-3$. In fact, the de Rham algebra of such a manifold is equivalent to one with non-vanishing differential in certain dimensions only, and for $r=2$, CN2015 implies that any simply connected closed 7-manifold is almost formal in the sense of [CKT2018], i.e., the $G_{2}$-structure is not needed in the simply connected case.

Our approach is to apply the homotopy transfer theorem to associate to an equivalence class of Poincaré DGCAs of Hodge type an equivalence class of $A_{\infty}$-algebras. We show that a simply connected Poincaré DGCA of Hodge type is equivalent to a certain finite dimensional Poincaré DGCA of Hodge type, so that the associated $A_{\infty}$-structure is simpler to describe. For instance, in the case of a $(r-1)$-connected $(r>1)$ Poincaré DGCA of Hodge type of dimension at most $5 r-3$, the resulting $A_{\infty}$-algebra is an $A_{3}$-algebra and thus determined by its cohomology algebra $H^{*}\left(\mathcal{A}^{*}\right)$ and the distinguished Hochschild cohomology class $\left[\mu_{3}\right] \in \operatorname{Hoch}^{3,-1}\left(H^{*}\left(\mathcal{A}^{*}\right), H^{*}\left(\mathcal{A}^{*}\right)\right) \subseteq$ $\operatorname{Hoch}^{2}\left(H^{*}\left(\mathcal{A}^{*}\right), H^{*}\left(\mathcal{A}^{*}\right)\right)$. Moreover, $\mu_{3}$ is actually a Harrison cocycle. Since in characteristic zero the natural morphism from Harrison cohomology to Hochschild cohomology of a DGCA is injective Barr1968, this means that $\left[\mu_{3}\right]$ is equivalently a Harrison cohomology class. As it is known that two simply-connected spaces are rationally homotopy equivalent if and only their de Rham complexes are $A_{\infty}$-quasi-isomorphic [Valette2012, Theorem 8], Kadeishvili2009, this implies that the rational homotopy type of a $(r-1)$ connected $(r>1)$ closed manifold $X$ of dimension at most $5 r-3$ is completely determined by $H^{*}(X)$ and $\left[\mu_{3}\right] \in \operatorname{Harr}^{3,-1}\left(H^{*}(X), H^{*}(X)\right)$. Relating this to the results in [CN2015], this means that $\left[\mu_{3}\right]$ is the Harrison cohomology interpretation of the Bianchi-Massey tensor. More precisely, the Bianchi-Massey tensor appears via the Harrison-to-André-Quillen spectral sequence for $\mathcal{A}^{*}$ Barr1968, Loday1992.

The condition of non-simply connectedness may be weakened to the existence of a Riemannian metric on $M$ for which all harmonic 1-forms are parallel. This is the case, for instance, if $M$ carries a metric of nonnegative Ricci curvature and, in particular, for $G_{2}$-manifolds as these are necessarily Ricci flat. Namely, by a generalization of the Cheeger-Gromoll splitting theorem (Theorem 5.1) it follows that in this case, the de Rham algebra is equivalent to a polynomial algebra $\mathcal{Q}^{*}\left[t_{1}, \ldots, t_{k}\right]$ with $k=b^{1}(M)\left|t_{i}\right|=1$ 
where $\mathcal{Q}^{*}$ is a Poincaré DGCA of lower degree (Proposition 5.3). In consequence, we show 7-manifolds with nonnegative Ricci curvature and positive $b^{1}$ must be formal; in particular, this is the case for $G_{2}$-manifold whose holonomy is properly contained in $G_{2}$. Note that the question of the formality of $G_{2}$-manifolds is still open.

Our paper is organized as follows. In Section 2 we define and study Poincaré DGCA's admitting a Hodge type decomposition. In Section 3 we define the small quotient algebra of such DGCA's of Hodge type, a Poincaré DGCA equivalent to the original one, which is finite dimensional if the DGCA is simply connected. In Section 4 we utilize the homotopy transfer theorem and related techniques to show that the rational homotopy type of a $(r-1)$ connected Poincaré DGCA $\mathcal{A}^{*}$ of dimension $n \leq 5 r-3$ is determined by $H^{*}\left(\mathcal{A}^{*}\right)$ and $\left[\mu_{3}\right] \in \operatorname{Harr}^{3,-1}\left(H^{*}\left(\mathcal{A}^{*}\right), H^{*}\left(\mathcal{A}^{*}\right)\right)$. In Section 5 we generalize the discussion from simply connected manifolds to those which admit a Riemannian metric all of whose harmonic 1-forms are parallel. Finally, in Appendix A we prove a few results on higher Massey products on connected Poincaré DGCAs.

Conventions and notations.

- In this paper we consider only DGCAs over a fixed field $\mathbb{F}$ of characteristic 0 .

- For a DGCA $\left(\mathcal{A}^{*}, d\right)$ we denote by $\mathcal{A}_{d}^{*}$ the subspace of cocycles in $\mathcal{A}^{*}$ and by $H^{*}\left(\mathcal{A}^{*}\right)$ the cohomology of $\left(\mathcal{A}^{*}, d\right)$. The Betti numbers of $\mathcal{A}^{*}$ are defined as $b^{k}\left(\mathcal{A}^{*}\right):=\operatorname{dim} \mathcal{H}^{k}\left(\mathcal{A}^{*}\right)$ or simply by $b^{k}$.

\section{Poincaré DGCAs of Hodge type}

In this section we recall the definition of a Poincaré DGCA over $\mathbb{F}$ (Definition 2.1, cf. CN2015, Definition 2.7]). Then we define Hodge type decompositions of Poincaré DGCAs (Definition 2.2) $\mathcal{A}^{*}$, provide examples and investigate their properties.

Definition 2.1. (cf. CN2015, Definition 2.7])

(1) A Poincaré-algebra of degree $n$ is a finite dimensional graded commutative algebra $H^{*}=\bigoplus_{k=0}^{n} H^{k}$ together with an element $\int \in\left(H^{n}\right)^{\vee}$, where the latter denotes the dual space of $H^{n}$, such that the pairing

$$
\left\langle\alpha^{k}, \beta^{l}\right\rangle:= \begin{cases}\int \alpha^{k} \cdot \beta^{l} & \text { if } k+l=n \\ 0 & \text { else }\end{cases}
$$

is non-degenerate, i.e., $\left\langle\alpha, H^{*}\right\rangle=0$ iff $\alpha=0$.

(2) A Poincaré-DGCA of degree $n$ is a $\operatorname{DGCA}\left(\mathcal{A}^{*}, d\right)$ with $\mathcal{A}^{*}=\bigoplus_{k=0}^{n} \mathcal{A}^{k}$ whose cohomology ring $H^{*}:=H^{*}\left(\mathcal{A}^{*}\right)$ is a Poincaré-algebra of degree $n$. 
Note that in [CN2015, Definition 2.7], the degree of a Poincaré-algebra is called the dimension, but as later we wish to consider the dimension of $\mathcal{A}^{*}$ as a graded vector space, the notion of degree seems more appropriate.

We begin our discussion by introducing some general terminology on pairings on a graded vector space. A graded vector space of degree $n$ is a vector space of the form $V^{*}=\bigoplus_{k=0}^{n} V^{k}$, and a bilinear pairing $\langle-,-\rangle$ on such a $V$ is called graded symmetric, if $\left\langle V^{k}, V^{l}\right\rangle=0$ for $k+l \neq n$ or, equivalently, if the map $\langle-,-\rangle: V^{*} \otimes V^{*} \rightarrow \mathbb{F}$ has degree $-n$, and factors through $\operatorname{Sym}^{2}(V)$, i.e., $\left\langle\alpha^{k}, \beta^{l}\right\rangle=(-1)^{k l}\left\langle\beta^{l}, \alpha^{k}\right\rangle$ for any $\alpha^{k} \in V^{k}$ and any $\beta^{l} \in V^{l}$. As usual, we define the orthogonal complement of a (graded) subspace $W^{*} \subseteq V^{*}$ as

$$
W_{\perp}^{*}:=\left\{\alpha \in V^{*} \mid\left\langle\alpha, W^{*}\right\rangle=0\right\},
$$

and call $V_{\perp}^{*}$ the null-space of $V^{*}$. A subspace $W^{*} \subseteq V^{*}$ is called nondegenerate if $W^{*} \cap W_{\perp}^{*}=0$. We call the pairing non-degenerate if $V^{*}$ is non-degenerate, i.e., iff $V_{\perp}^{*}=0$. On the quotient $Q^{*}:=V^{*} / V_{\perp}^{*}$, there is a unique non-degenerate pairing $\langle-,-\rangle_{Q^{*}}$ such that

$$
\langle\pi(\alpha), \pi(\beta)\rangle_{Q^{*}}=\langle\alpha, \beta\rangle,
$$

where $\pi: V^{*} \rightarrow Q^{*}$ is the canonical projection.

Let us now apply all of this to a Poincaré-DGCA $\mathcal{A}^{*}$ of degree $n$. The pairing $\langle-,-\rangle$ on $H^{*}\left(\mathcal{A}^{*}\right)$ induces a graded pairing, denoted by the same symbol, defined as

$$
\left\langle\alpha^{k}, \beta^{l}\right\rangle= \begin{cases}\int\left[\alpha^{k} \cdot \beta^{l}\right] & \text { if } k+l=n \\ 0 & \text { else. }\end{cases}
$$

where [.] stands for the projection $\mathcal{A}^{n}=\mathcal{A}_{d}^{n} \rightarrow H^{n}\left(\mathcal{A}^{*}\right)$. It immediately follows that $\langle-,-\rangle$ satisfies

$$
\begin{array}{ll}
\left\langle\alpha^{k}, \beta^{l}\right\rangle & =(-1)^{k l}\left\langle\beta^{l}, \alpha^{k}\right\rangle, \\
\left\langle\alpha^{k} \cdot \beta^{l}, \gamma^{r}\right\rangle & =\left\langle\alpha^{k}, \beta^{l} \cdot \gamma^{r}\right\rangle, \\
\left\langle d \alpha^{k}, \beta^{l}\right\rangle & =(-1)^{k+1}\left\langle\alpha^{k}, d \beta^{l}\right\rangle .
\end{array}
$$

Poincaré-DGCAs are examples of cyclic $A_{\infty}$-algebras that have been introduced by Kontsevich [Kontsevich1994]. The cyclic DGCA $\left(\Omega^{*}(M), d\right)$, $H_{d R}^{*}(M)$ have been considered in other papers, e.g. Hajek2018.

Definition 2.2. Let $\left(\mathcal{A}^{*},\langle-,-\rangle\right)$ be a Poincaré-DGCA.

(1) We call $\mathcal{A}^{*}$ non-degenerate if the pairing $\langle-,-\rangle$ from (2.2) is nondegenerate.

(2) A harmonic subspace is a subspace $\mathcal{H}^{*} \subseteq \mathcal{A}_{d}^{*}$ complementary to $d \mathcal{A}^{*}$.

(3) A splitting map of $\mathcal{A}^{*}$ is a splitting $\imath: H^{*} \rightarrow \mathcal{A}_{d}^{*}$ which splits the short exact sequence

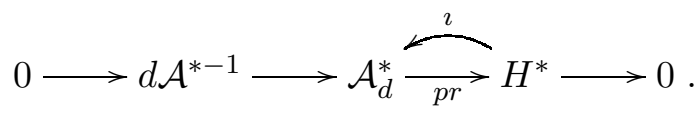


(4) A Hodge type decomposition is a direct sum decomposition of the form

$$
\mathcal{A}^{*}=d \mathcal{A}^{*-1} \oplus \mathcal{H}^{*} \oplus \mathcal{B}^{*},
$$

where $\mathcal{H}^{*}$ is a harmonic subspace and such that

$$
\left\langle\mathcal{H}^{*} \oplus \mathcal{B}^{*}, \mathcal{B}^{*}\right\rangle=0
$$

(5) If $\mathcal{A}^{*}$ admits a Hodge type decomposition, then we call $\mathcal{A}^{*}$ a Poincaré $D G C A$ of Hodge type.

Remark 2.3. Evidently there is a one-to-one correspondence of harmonic subspaces $\mathcal{H}^{*} \subseteq \mathcal{A}_{d}^{*}$ and splitting maps of $\mathcal{A}^{*}$, as the image of a splitting map is a harmonic subspace. As $\imath: H^{*} \rightarrow \mathcal{H}^{*}$ preserves the pairing $\langle-,-\rangle$, it follows that the graded subspace $\mathcal{H}^{*} \subseteq \mathcal{A}^{*}$ is non-degenerate.

Remark 2.4. By (2.3), $\mathcal{A}_{\perp}^{*} \subseteq \mathcal{A}^{*}$ is a $d$-invariant ideal, whence there is an exact sequence of DGCA

$$
0 \longrightarrow \mathcal{A}_{\perp}^{*} \longrightarrow \mathcal{A}^{*} \longrightarrow \mathcal{Q}^{*} \longrightarrow 0,
$$

where $\mathcal{Q}^{*}$ is by definition the quotient $\mathcal{A}^{*} / \mathcal{A}_{\perp}^{*}$.

Example 2.5. The prototypical example of a Poincaré algebra of degree $n$ of Hodge type (which motivates our terminology) is the de Rham algebra $\left(\Omega^{*}(M), d\right)$ of a closed smooth oriented manifold $M$, with $\int$ given by the integration of $n$-forms. The Hodge decomposition w.r.t. some Riemannian metric $g$ on $M$

$$
\Omega^{*}(M)=d \Omega^{*-1}(M) \oplus \mathcal{H}^{*}(M) \oplus d^{*} \Omega^{*+1}(M)
$$

yields a Hodge type decomposition in the sense of Definition [2.2, and the space $\mathcal{H}^{*}(M)$ of $\triangle_{g}$-harmonic forms is a harmonic subspace in the above sense. As we shall see in Remark 2.6 below, any other harmonic subspace $\mathcal{H}^{*} \subseteq \Omega_{d}^{*}(M)$ will give rise to a Hodge type decomposition as well.

Remark 2.6. If $\left(\mathcal{A}^{*}, d\right)$ is a Poincaré-DGCA admitting a Hodge-type decomposition, then every harmonic subspace $\mathcal{H}^{*} \subseteq \mathcal{A}_{d}^{*}$ may occur as summand of a Hodge-type decomposition (2.4). Namely, given a Hodge type decomposition (2.4) and a harmonic subspace $\hat{\mathcal{H}}^{*} \subseteq \mathcal{A}_{d}^{*}$, from the identity $\mathcal{H}^{*} \oplus d \mathcal{A}^{*-1}=\hat{\mathcal{H}}^{*} \oplus d \mathcal{A}^{*-1}$ it follows that there is a linear map $\alpha: \mathcal{H}^{*} \rightarrow$ $d \mathcal{A}^{*-1}$ such that

$$
\hat{\mathcal{H}}^{*}=\left\{v+\alpha(v) \mid v \in \mathcal{H}^{*}\right\} .
$$

Letting $\hat{\mathcal{B}}^{*}:=\left\{x-\alpha^{\dagger}(x)-\frac{1}{2} \alpha \alpha^{\dagger}(x) \mid x \in \mathcal{B}^{*}\right\}$, where $\alpha^{\dagger}: \mathcal{B}^{*} \rightarrow \mathcal{H}^{*}$ is the unique map satisfying $\left\langle\alpha^{\dagger}(x), v\right\rangle=\langle x, \alpha(v)\rangle \quad$ for all $x \in \mathcal{B}^{*}, v \in \mathcal{H}^{*}$, the decomposition $\mathcal{A}^{*}=\hat{\mathcal{H}}^{*} \oplus d \mathcal{A}^{*-1} \oplus \hat{\mathcal{B}}^{*}$ is a Hodge type decomposition.

Given a Hodge-type decomposition (2.4), it follows that the restriction $d: \mathcal{B}^{*} \rightarrow d \mathcal{A}^{*}$ is both injective and surjective and hence has an inverse 
$d^{-}: d \mathcal{A}^{*} \rightarrow \mathcal{B}^{*}$. We may extend $d^{-}$to all of $\mathcal{A}^{*}$ by defining $d_{\mid \mathcal{H}^{*} \oplus \mathcal{B}^{*}}^{-}=0$. Thus, $\left(d^{-}\right)^{2}=0$, and

$$
d d^{-} d=d, \quad d^{-} d d^{-}=d^{-} .
$$

It follows that the projections in (2.4) are given by

$$
p r_{\mathcal{H}^{*}}=1-\left[d, d^{-}\right], \quad p r_{d \mathcal{A}^{*-1}}=d d^{-}, \quad p r_{\mathcal{B}^{*}=d^{-} \mathcal{A}^{*+1}}=d^{-} d,
$$

where $\left[d, d^{-}\right]=d d^{-}+d^{-} d$ is the super-commutator. Therefore, (2.4) may be written as

$$
\mathcal{A}^{*}=d \mathcal{A}^{*-1} \oplus \mathcal{H}^{*} \oplus d^{-} \mathcal{A}^{*+1}=d d^{-} \mathcal{A}^{*} \oplus \mathcal{H}^{*} \oplus d^{-} d \mathcal{A}^{*},
$$

and setting $\mathcal{A}_{d^{-}}^{*}:=\operatorname{ker} d^{-}=\mathcal{H}^{*} \oplus d^{-} \mathcal{A}^{*},(2.5)$ implies

$$
\left\langle\mathcal{A}_{d}^{*}, d \mathcal{A}^{*-1}\right\rangle=\left\langle\mathcal{A}_{d^{-}}^{*}, d^{-} \mathcal{A}^{*+1}\right\rangle=0 .
$$

We shall call elements in $\mathcal{A}_{d^{-}}^{*}$ and $d^{-} \mathcal{A}^{*+1} d^{-}$-closed and $d^{-}$-exact, respectively.

Recall that a DGA over $\mathbb{F}$ is called connected if $H^{0}\left(\mathcal{A}^{*}\right) \cong \mathbb{F}$ and $r$ connected if it is connected and $H^{k}\left(\mathcal{A}^{*}\right)=0$ for $k=1, \ldots, r$. A 1-connected DGA is also called simply connected.

Remark 2.7. (1) For $\mathcal{A}^{*}=\left(\Omega^{*}(M), d\right)$ for a closed oriented manifold $M$ with its Hodge decomposition w.r.t. a Riemannian metric $g$ (cf. Example 2.5) the map $d^{-}$in (2.10) is not the codifferential $d^{*}$ in (2.7). In particular, the supercommutator $\triangle_{g}=\left[d, d^{*}\right]$ is not a projector. Rather, it is not hard to see that $d^{-}$and $d^{*}$ are related by the formula $d^{*}=\triangle_{g} d^{-}$.

(2) Note that $\left(\Omega^{*}(M), d\right)$ is connected iff $M$ is connected. However, the $r$-connectedness of $\left(\Omega^{*}(M), d\right)$, i.e., the vanishing of $b^{k}(M), 1 \leq k \leq$ $r$, is a weaker notion than the $r$-connectedness of $M$, as the latter means that the homotopy groups $\pi_{k}(M)$ are trivial for $k \leq r$ (which is stronger than the vanishing of $\left.b^{k}(M), 1 \leq k \leq r\right)$.

(3) If the harmonic subspace $\mathcal{H}^{*}$ happens to be a subalgebra of $\mathcal{A}^{*}$, i.e., if the product of two harmonic elements is harmonic, then $\left(\mathcal{A}^{*}, d\right)$ is a formal DGCA.

(4) A Riemannian manifold $(M, g)$ for which the product of $\triangle_{g}$-harmonic forms is $\triangle_{g}$-harmonic is called geometrically formal. It is known that compact symmetric spaces are geometrically formal Sullivan1975, but in general, there are strong topological obstructions for $M$ to admit a geometrically formal Riemannian metric, see Kotschick2001 for details and classification results of low dimensional geometrically formal Riemannian manifolds. The relation between the Hodge decomposition of the de Rham complex on a closed Riemannian manifold $M$ and the minimal model of $M$ in the case $b^{1}(M)=0$ has been proposed by Sullivan Sullivan1977, Sullivan1975. The 
Hodge decomposition is also important in the proof of the formality of closed Kähler manifolds by Deligne-Griffiths-Morgan-Sullivan [DGMS1975].

(5) We will be only interested in connected Poincaré DGCAs. In this case the product in cohomology $H^{0}\left(\mathcal{A}^{*}\right) \otimes H^{k}\left(\mathcal{A}^{*}\right) \rightarrow H^{k}\left(\mathcal{A}^{*}\right)$ is easily seen to be multiplication by scalars on the $\mathbb{F}$-vector space $H^{k}\left(\mathcal{A}^{*}\right)$.

Lemma 2.8. If the Poincaré DGCA $\mathcal{A}^{*}$ admits a Hodge type decomposition, then the differential ideal $\mathcal{A}_{\perp}^{*}$ is invariant under $d^{-}$, and has the decomposition

$$
\mathcal{A}_{\perp}^{*}=d d^{-} \mathcal{A}_{\perp}^{*} \oplus d^{-} d \mathcal{A}_{\perp}^{*} .
$$

In particular, $\left(\mathcal{A}_{\perp}^{*}, d\right)$ has trivial cohomology.

Proof. We begin by showing that the restriction of the harmonic projector $p r_{\mathcal{H}^{*}}$ to $\mathcal{A}_{\perp}^{*}$ is zero. To see this, let $\alpha^{k} \in \mathcal{A}_{\perp}^{k}$ and write

$$
\alpha^{k}=\operatorname{pr}_{\mathcal{H}^{*}}\left(\alpha^{k}\right)+d d^{-} \alpha^{k}+d^{-} d \alpha^{k}
$$

Then, for any $\beta^{n-k} \in \mathcal{H}^{n-k}$, we have

$$
0=\left\langle\alpha^{k}, \beta^{n-k}\right\rangle=\left\langle p r_{\mathcal{H}^{*}}\left(\alpha^{k}\right), \beta^{n-k}\right\rangle,
$$

as $\mathcal{H}^{n-k} \subseteq \mathcal{A}^{n-k}$ and by the orthogonality relations (2.5)-(2.11). Since the pairing $\langle-,-\rangle$ is nondegenerate on $\mathcal{H}^{*}\left(\operatorname{Remark}[2.3)\right.$, this implies $\operatorname{pr}_{\mathcal{H}^{*}}\left(\alpha^{k}\right)=$ 0 . Now we can show that $\mathcal{A}_{\perp}^{*}$ is stable with respect to both projections $d d^{-}$ and $d^{-} d$. As we have shown that

$$
\alpha^{k}=d d^{-} \alpha^{k}+d^{-} d \alpha^{k}
$$

for any $\alpha^{k} \in \mathcal{A}_{\perp}^{k}$, it will suffice to show that $d^{-} d \alpha^{k} \in \mathcal{A}_{\perp}^{k}$. By the orthogonality relations (2.5) and by the decomposition (2.4), we only need to show that $\left\langle d^{-} d \alpha^{k}, d d^{-} \beta^{n-k}\right\rangle=0$. We compute

$$
\begin{aligned}
\left\langle d^{-} d \alpha^{k}, d d^{-} \beta^{n-k}\right\rangle & =(-1)^{k+1}\left\langle d d^{-} d \alpha^{k}, d^{-} \beta^{n-k}\right\rangle \\
& =(-1)^{k+1}\left\langle d \alpha^{k}, d^{-} \beta^{n-k}\right\rangle \\
& =\left\langle\alpha^{k}, d d^{-} \beta^{n-k}\right\rangle=0
\end{aligned}
$$

where we used $d d^{-} d=d$ and the fact that $d d^{-} \beta^{n-k} \in \mathcal{A}^{n-k}$ by (2.8] 2.9]). This shows that we have the direct sum decomposition

$$
\mathcal{A}_{\perp}^{*}=d d^{-} \mathcal{A}_{\perp}^{*} \oplus d^{-} d \mathcal{A}_{\perp}^{*} .
$$

Finally, we show that $d^{-} \mathcal{A}_{\perp}^{k} \subseteq \mathcal{A}_{\perp}^{k-1}$. To see this, let $\alpha^{k} \in \mathcal{A}_{\perp}^{k}$. As $d^{-} \mathcal{A}_{\perp}^{k}=$ $d^{-} d d^{-} \mathcal{A}_{\perp}^{k}$, we want to show that $\left\langle d^{-} d d^{-} \alpha^{k}, \beta^{n-k+1}\right\rangle=0$ for any $\beta^{n-k+1} \in$ $\mathcal{A}^{n-k+1}$. As $d^{-} d d^{-} \alpha^{k} \in d^{-} \mathcal{A}^{k-1}$, by the orthogonality relations (2.5) and from the direct sum decomposition (2.4) we see that it is not restrictive to assume $\beta^{n-k+1}=d d^{-} \gamma^{n-k+1}$, with $\gamma^{n-k+1} \in \mathcal{A}^{n-k+1}$. We have

$$
\left\langle d^{-} d d^{-} \alpha^{k}, d d^{-} \gamma^{n-k+1}\right\rangle=(-1)^{k}\left\langle d d^{-} d d^{-} \alpha^{k}, d^{-} \gamma^{n-k+1}\right\rangle=0
$$


where we used that $\mathcal{A}_{\perp}^{*}$ is stable with respect to $d d^{-}$. As $\operatorname{Id}_{\mathcal{A}_{\perp}^{*}}=\left[d, d^{-}\right]$ with $d^{-}$a degree -1 endomorphism of $\mathcal{A}_{\perp}^{*}$, we see that the identity of $\mathcal{A}_{\perp}^{*}$ is null-homotopic and so $\left(\mathcal{A}_{\perp}^{*}, d\right)$ is an acyclic complex.

Corollary 2.9. If $\mathcal{A}^{*}$ is of Hodge type, then the projection $\pi: \mathcal{A}^{*} \rightarrow \mathcal{Q}^{*}$, where $\mathcal{Q}^{*}=\mathcal{A}^{*} / \mathcal{A}_{\perp}^{*}$, is a quasi-isomorphism.

Remark 2.10. As the induced pairing on $\mathcal{Q}^{*}$ is nondegenerate by construction, Corollary 2.9 implies that every Hodge type Poincaré DGCA is equivalent to a nondegenerate one. This is actually true even without the assumption that the Poincaré-DGCA $\mathcal{A}^{*}$ is of Hodge type, but the general proof is much more involved: see [LS2007, Theorem 1.1].

Example 2.11. We conclude this section with an example of a Poincaré DGCA admitting no Hodge type decomposition. Let $\mathcal{A}^{*}$ be the graded vector space on linear generators $\left\{x_{0}, x_{2}, x_{3}, x_{4}\right\}$ with $\operatorname{deg}\left(x_{i}\right)=i$. That is $\mathcal{A}^{k}$ is 1 -dimensional for $k \in\{0,2,3,4\}$ and 0 -dimensional for any other value of $k$. Introduce a DGCA structure on $\mathcal{A}^{*}$ by declaring the product and the differential on the generators as follows:

- the only non-zero products are $x_{0} \cdot x_{i}=x_{i} \cdot x_{0}=x_{i}$ and $x_{2} \cdot x_{2}=x_{4}$;

- the only non-zero differential is $d x_{2}=x_{3}$.

The cohomology of $\mathcal{A}^{*}$ is the graded algebra on linear generators $\left[x_{0}\right]$ and $\left[x_{4}\right]$ with the products $\left[x_{0}\right] \cdot\left[x_{0}\right]=\left[x_{0}\right],\left[x_{0}\right] \cdot\left[x_{4}\right]=\left[x_{4}\right] \cdot\left[x_{0}\right]=\left[x_{4}\right]$ and $\left[x_{4}\right] \cdot\left[x_{4}\right]=0$. Therefore, the only harmonic subspace is $\mathcal{H}^{*}=\operatorname{span}\left(x_{0}, x_{4}\right)$. The linear functional $\int: H^{4}\left(\mathcal{A}^{*}\right) \rightarrow \mathbb{F}$ given by $\int\left[x_{4}\right]=1$ makes $\mathcal{A}^{*}$ a degree 4 Poincaré DGCA. The $d$-invariant ideal $\left(\mathcal{A}_{\perp}^{*}, d\right)$ consists of $\mathcal{A}^{3}$ in degree 3 with the zero differential and so it is manifestly not acyclic. Therefore, $\left(\mathcal{A}^{*},\langle-,-\rangle\right)$ cannot have a Hodge type decompositions by Lemma 2.8 .

Remark 2.12. Observe that in Example 2.11 $\mathcal{H}^{*}$ is a subalgebra of $\mathcal{A}^{*}$, whence the inclusion $\mathcal{H}^{*} \hookrightarrow \mathcal{A}^{*}$ is a quasi-isomorphism, and evidently $\mathcal{H}^{*}$ is of Hodge type. That is, being of Hodge type is a property not preserved by quasi-isomorphisms.

Remark 2.13. In CFL2015, Lemma 11.1] Cieliebak-Fukaya-Latschev proved that a finite dimensional non-degenerate Poincare DGCA always admits a Hodge type decomposition.

\section{Small quotient algebras of Poincaré DGCAs of Hodge type}

In this section we define the small algebra of a Poincaré DGCA of Hodge type $\mathcal{A}^{*}$ and the small quotient algebra of $\mathcal{A}^{*}$ (Definition 3.2). These algebras are DGCAs equivalent to $\mathcal{A}^{*}$. As a result we show that any simply connected Poincaré-DGCA of Hodge type is equivalent to a non-degenerate finite dimensional Poincaré-DGCA (Corollary 3.5). We investigate the equivalence class of a $(r-1)$ connected $(r>1)$ Poincaré DGCA (Theorem 3.10, Corollary 3.11) and compare our results with some known results (Remark 3.12). 
Definition 3.1. Let $\mathcal{A}^{*}$ be a Poincaré DGCA with a Hodge-type decomposition (2.10). A $\mathcal{H}^{*}$-subalgebra is a DG-subalgebra of $\mathcal{A}^{*}$ which is $d^{-}$-invariant and contains $\mathcal{H}^{*}$.

For any $\mathcal{H}^{*}$-subalgebra $\mathcal{C}^{*}$ of $\mathcal{A}^{*}$, the restriction of the inner product of $\mathcal{A}^{*}$ to $\mathcal{C}^{*}$ makes $\mathcal{C}^{*}$ a degree $n$ Poincaré DGCA and the Hodge-type decomposition of $\mathcal{A}^{*}$ induces a Hodge-type decomposition

$$
\mathcal{C}^{*}=\mathcal{H}^{*} \oplus d \mathcal{C}^{*} \oplus d^{-} \mathcal{C}^{*}
$$

as $\mathcal{C}^{*}$ contains $\mathcal{H}^{*}$ and is invariant under the projections (2.9). In particular, the inclusion $\left(\mathcal{C}^{*}, d\right) \hookrightarrow\left(\mathcal{A}^{*}, d\right)$ is a quasi-isomorphism. Taking the quotient $\mathcal{Q}^{*}\left(\mathcal{C}^{*}\right):=\mathcal{C}^{*} / \mathcal{C}_{\perp}^{*}$, Corollary 2.9 and Lemma 2.8 imply that $\mathcal{Q}^{*}\left(\mathcal{C}^{*}\right)$ is a nondegenerate Poincaré algebra of Hodge type, and the inclusion and projection maps

$$
\mathcal{A}^{*} \longleftarrow \mathcal{C}^{*} \longrightarrow \mathcal{Q}^{*}\left(\mathcal{C}^{*}\right)
$$

are quasi-isomorphisms, so that $\mathcal{A}^{*}$ is equivalent to $\mathcal{Q}^{*}\left(\mathcal{C}^{*}\right)$.

Definition 3.2. Let $\mathcal{A}^{*}=d \mathcal{A}^{*} \oplus \mathcal{H} \oplus d^{-} \mathcal{A}^{*}$ be a Poincaré DGCA with a Hodge type decomposition. The small algebra of $\mathcal{A}^{*}$, denoted by $\mathcal{A}_{\text {small }}^{*}$ or $\mathcal{A}_{\text {small }}^{*}\left(\mathcal{H}^{*}\right)$ is the (unique) smallest $\mathcal{H}^{*}$-subalgebra of $\mathcal{A}^{*}$. The small quotient algebra of $\mathcal{A}^{*}$ is the quotient DGCA $\mathcal{Q}_{\text {small }}^{*}\left(\mathcal{H}^{*}\right)$ or $\mathcal{Q}_{\text {small }}^{*}:=\mathcal{Q}^{*}\left(\mathcal{A}_{\text {small }}^{*}\right)=$ $\mathcal{A}_{\mathrm{small}}^{*} /\left(\mathcal{A}_{\mathrm{small}}^{*}\right)_{\perp}$.

Clearly, the smallest algebra is well defined as $\mathcal{A}^{*}$ is a $\mathcal{H}^{*}$-subalgebras and the intersection of an arbitrary family of $\mathcal{H}^{*}$-subalgebras is a $\mathcal{H}^{*}$-subalgebra, so that $\mathcal{A}_{\text {small }}^{*}$ is the intersection of all $\mathcal{H}^{*}$-subalgebras of $\mathcal{A}^{*}$. We shall, however, give a more accessible description of $\mathcal{A}_{\text {small }}^{*}$.

For a $\mathcal{H}^{*}$-subalgebra $\mathcal{C}^{*}$ of $\mathcal{A}^{*}$ we denote by $\mathcal{C}^{i, j} \subseteq \mathcal{C}^{i+j}$ the image of the multiplication $\mathcal{C}^{i} \otimes \mathcal{C}^{j} \rightarrow \mathcal{C}^{i+j}$.

Proposition 3.3. Let $\mathcal{A}^{*}$ be a simply connected Poincaré DGCA endowed with a Hodge type decomposition. Then its small algebra $\mathcal{A}_{\mathrm{small}}^{*}$ is defined by the recursive formula

$$
\left\{\begin{aligned}
\mathcal{A}_{\mathrm{small}}^{0}= & \mathbb{F} \cdot 1_{\mathcal{A}} \\
\mathcal{A}_{\mathrm{small}}^{1}= & 0 \\
\mathcal{A}_{\mathrm{small}}^{k}= & \mathcal{H}^{k} \oplus d d^{-} \operatorname{span}\left\{\mathcal{A}_{\mathrm{small}}^{l_{1}, l_{2}}, l_{1}, l_{2} \geq 2, l_{1}+l_{2}=k\right\} \\
& \oplus d^{-} \operatorname{span}\left\{\mathcal{A}_{\mathrm{small}}^{l_{1}, l_{2}}, l_{1}, l_{2} \geq 2, l_{1}+l_{2}=k+1\right\} ; \quad k \geq 2
\end{aligned}\right.
$$

In particular $\mathcal{A}_{\mathrm{small}}^{*}$ is finite dimensional.

Proof. Let us denote by $\mathcal{C}^{*}$ the algebra defined by the recursion (3.2). Thus, our aim is to show that $\mathcal{C}^{*}=\mathcal{A}_{\text {small }}^{*}$.

It is immediate from the definition of $\mathcal{C}^{*}$ and from the identity $d^{-} d d^{-}=d^{-}$ that $\mathcal{H}^{*} \subseteq \mathcal{C}^{*}$ and $d \mathcal{C}^{k} \subseteq \mathcal{C}^{k+1}$ and $d^{-} \mathcal{C}^{k} \subseteq \mathcal{C}^{k-1}$. To see that $\mathcal{C}^{*}$ is closed under multiplication, we need to show that, if $\alpha^{k} \in \mathcal{C}^{k}$ and $\beta^{l} \in \mathcal{C}^{l}$, then 
$\alpha^{k} \cdot \beta^{l} \in \mathcal{C}^{k+l}$. If $k \leq 1$ or $l \leq 1$ there is nothing to be proven. So let us assume $k, l \geq 2$. By equations (3.1), (2.9) we have

$$
\alpha^{k} \cdot \beta^{l}=p r_{\mathcal{H}^{*}}\left(\alpha^{k} \cdot \beta^{l}\right)+d d^{-}\left(\alpha^{k} \cdot \beta^{l}\right)+d^{-}\left(d \alpha^{k} \cdot \beta^{l}\right)+(-1)^{k} d^{-}\left(\alpha^{k} \cdot d \beta^{l}\right),
$$

and the right-hand side manifestly belongs to $\mathcal{C}^{k+l}$, as $\mathcal{C}^{*}$ is $d$-closed so that $d \alpha^{k}, d \beta^{l} \in \mathcal{C}^{*}$.

Conversely, it follows by induction of $k$ that $\mathcal{C}^{k} \subseteq \mathcal{A}_{\text {small }}^{k}$. Indeed, for $k=0,1$ this is obvious, and if $k \geq 2$, then it is evident that any $\mathcal{H}^{*}$ subalgebra containing $\mathcal{C}^{l}$ for $l<k$ also must contain $\mathcal{C}^{k}$ by (3.2).

Remark 3.4. It is worth noticing that the proof of Proposition 3.3 does not use the existence of a pairing on $\mathcal{A}^{*}$ (and so in particular the existence of a Hodge-type decomposition) nor the graded commutativity of the multiplication. That is, Proposition 3.3 actually shows that a simply connected DGA $\mathcal{A}^{*}$ concentrated in degrees $[0, n]$ and with finite dimensional cohomology is always equivalent to a finite-dimensional DGA $\mathcal{A}_{\mathrm{small}}^{*}$.

As $\mathcal{Q}_{\text {small }}$ is equivalent to $\mathcal{A}^{*}$, we have thus shown the following.

Corollary 3.5. Let $\mathcal{A}^{*}$ be a simply connected Poincaré-DGCA of degree $n$ and of Hodge type. Then $\mathcal{A}^{*}$ is equivalent to a finite dimensional nondegenerate Poincaré-DGCA of degree $n$ and of Hodge type.

Remark 3.6. The result of Corollary 3.5 holds more generally without the assumption that the Poincaré-DGCA $\mathcal{A}^{*}$ is of Hodge type. Namely, one can apply Theorem 1.1 from [LS2007] to the small algebra $\mathcal{A}_{\text {small }}^{*}$ to get a finite dimensional non-degenerate Poincaré-DGCA $\widetilde{\mathcal{A}_{\text {small }}^{*}}$ of degree $n$. By Remark 2.13, this algebra will admit a Hodge type decomposition.

Given a connected Poincaré DGCA of degree $n \mathcal{A}^{*}$, with cohomology algebra $H^{*}:=H^{*}\left(\mathcal{A}^{*}\right)$, let $H_{+}^{*}:=\bigoplus_{k=1}^{n} H^{k}$.

Definition 3.7. A generating subspace is a graded subspace $H_{g e n}^{*} \subseteq H_{+}^{*}$ complementary to $H_{+}^{*} \cdot H_{+}^{*}$, i.e., there is a direct sum decomposition

$$
H_{+}^{*}=H_{g e n}^{*} \oplus\left(H_{+}^{*} \cdot H_{+}^{*}\right) .
$$

As $H^{*}$ is finite dimensional, $H_{\text {gen }}^{*}$ always exists and $H^{1} \subseteq H_{\text {gen }}^{*}$. Moreover, if $\mathcal{A}^{*}$ is $(r-1)$-connected, so that $H^{k}=0$ for $k=1, \ldots, r-1$, then $H^{k} \subseteq H_{\text {gen }}^{*}$ for $0<k \leq 2 r-1$. Induction on the degree easily implies that each element in $H_{+}^{*} \cdot H_{+}^{*}$ is spanned by products of elements in $H_{g e n}^{*}$. That is, $H_{\text {gen }}^{*}$ generates $H_{+}^{*}$ as an algebra.

Let $S^{*}\left(H_{\text {gen }}^{*}\right)$ be the graded symmetric tensor algebra of elements of $H_{\text {gen }}^{*}$. Multiplication induces a morphism of graded algebras $S^{*}\left(H_{\text {gen }}^{*}\right) \rightarrow H^{*}$, which is surjective, as $H_{\text {gen }}^{*}$ generates $H^{*}$ and $H^{0}=\mathbb{F}$ is spanned by the image of the identity in $S^{*}\left(H_{g e n}^{*}\right)$. Therefore, we obtain the exact sequence

$$
0 \longrightarrow K^{*} \longrightarrow S^{*}\left(H_{\text {gen }}^{*}\right) \longrightarrow H^{*} \longrightarrow 0
$$


where the kernel of the multiplication $K^{*} \subseteq S^{*}\left(H_{\text {gen }}^{*}\right)$ is an ideal. In fact, decomposing $S^{*}\left(H_{\text {gen }}^{*}\right)=\mathbb{F} \oplus H_{\text {gen }}^{*} \oplus S_{\geq 2}^{*}\left(H_{\text {gen }}^{*}\right)$, where $S_{>k}^{*}\left(H^{*}\right) \subseteq S^{*}\left(H^{*}\right)$ denotes the ideal of graded polynomials of degree at least $k$, multiplication maps the first two summands isomorphically to $H^{0}$ and $H_{\text {gen }}^{*}$, respectively, and $S_{\geq 2}^{*}\left(H_{\text {gen }}^{*}\right)$ to $H_{+} \cdot H_{+}$. Therefore, we may restrict (3.4) to a subsequence

$$
0 \longrightarrow K^{*} \longrightarrow S_{\geq 2}^{*}\left(H_{\text {gen }}^{*}\right) \longrightarrow H_{+}^{*} \cdot H_{+}^{*} \longrightarrow 0 .
$$

A linear map $\imath_{0}: H_{\text {gen }}^{*} \rightarrow \mathcal{A}_{d}^{*}$ will be called a partial splitting map if $p r \circ \imath_{0}$ is the inclusion of $H_{\text {gen }}^{*}$ into $H^{*}\left(\mathcal{A}^{*}\right)$ with $p r: \mathcal{A}_{d}^{*} \rightarrow H^{*}$ from Definition 2.2. As $H_{\text {gen }}^{*}$ is finite dimensional, a partial splitting map always exists, and we denote its image by $\mathcal{H}_{\text {gen }}^{*} \subseteq \mathcal{A}_{d}^{*}$. Then $\imath_{0}$ induces an algebra morphism $S^{*}\left(H_{\text {gen }}^{*}\right) \rightarrow \mathcal{A}_{d}^{*}$ which by abuse of notation we also denote by $\imath_{0}$, and $p r \circ \imath_{0}$ : $S^{*}\left(H_{\text {gen }}^{*}\right) \rightarrow H^{*}$ coincides with the multiplication map ". "from above. Observe that $\imath_{0}\left(S^{*}\left(H_{\text {gen }}^{*}\right)\right)$ is the algebra generated by $\mathcal{H}_{\text {gen }}^{*} \subseteq \mathcal{A}_{d}^{*}$.

Definition 3.8. Let $H_{\text {gen }}^{*} \subseteq H_{+}^{*}$ be a generating subspace and $\imath_{0}: H_{\text {gen }}^{*} \rightarrow$ $\mathcal{A}_{d}^{*}$ be a partial splitting map. We call a splitting map $\imath: H^{*}\left(\mathcal{A}^{*}\right) \rightarrow \mathcal{A}_{d}^{*}$ an extension of $\iota_{0}$ if $\imath_{\mid H_{\text {gen }}^{*}}=\iota_{0}$ and $\imath\left(H^{*}\left(\mathcal{A}^{*}\right)\right) \subseteq \iota_{0}\left(S^{*}\left(H_{\text {gen }}^{*}\right)\right)$. In this case, we say that the harmonic subspace $\mathcal{H}^{*}=\imath\left(H^{*}\left(\mathcal{A}^{*}\right)\right)$ is $\imath_{0}$-adapted; we call $\mathcal{H}_{\text {gen }}^{*}$ a generating harmonic space, and $\mathcal{H}^{*}=\imath\left(H^{*}\right)$ an extension of $\mathcal{H}_{\text {gen }}^{*}$.

Thus, we have the following commuting diagrams of short exact sequences
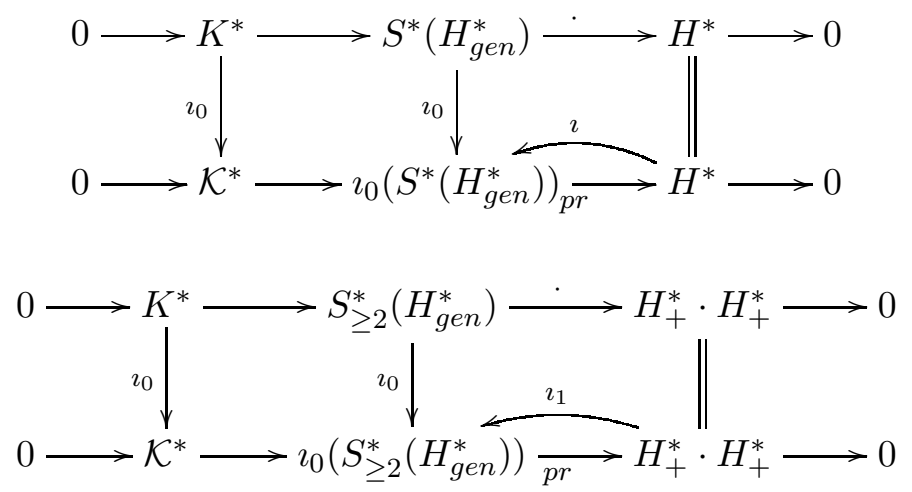

where the top rows are the sequence (3.4) and (3.5), respectively, and the vertical maps are surjective, and where $\mathcal{K}^{*}:=\iota_{0}\left(K^{*}\right) \subseteq \mathcal{A}^{*}$.

Lemma 3.9. There is a one-to-one correspondence between $\imath_{0}$-adapted splitting maps $\imath: H^{*} \rightarrow \mathcal{A}_{d}^{*}$ and splitting maps $\imath_{1}: H_{+}^{*} \cdot H_{+}^{*} \rightarrow \imath_{0}\left(S_{\geq 2}^{*}\left(H_{\text {gen }}^{*}\right)\right)$ of the exact sequence in the bottom row of (3.7). In particular, such a splitting map always exists.

Proof. Given a splitting map $\imath_{1}: H_{+}^{*} \cdot H_{+}^{*} \rightarrow \imath_{0}\left(S_{\geq 2}^{*}\left(H_{\text {gen }}^{*}\right)\right)$ of (3.5), it follows that $\imath:=\imath_{0} \oplus \imath_{1}: H^{*}=H_{g e n}^{*} \oplus H_{+}^{*} \cdot H_{+}^{*} \rightarrow \mathcal{A}_{d}^{*}$ (using the decomposition (3.3)) is a splitting of (3.4) whose image is contained in $\imath_{0}\left(S^{*}\left(H_{\text {gen }}^{*}\right)\right)$. Conversely, given a splitting $\imath: H^{*} \rightarrow \iota_{0}\left(S^{*}\left(H_{\text {gen }}^{*}\right)\right)$ of (3.4), its restriction to $H_{+}^{*}$. 
$H_{+}^{*}$ maps to $\imath_{0}\left(S_{\geq 2}^{*}\left(H_{\text {gen }}^{*}\right)\right)$ and hence yields a splitting map of (3.5). The existence of such a splitting follows as $H_{+}^{*} \cdot H_{+}^{*}$ is finite dimensional.

By definition, if $\mathcal{A}^{*}$ is $(r-1)$-connected, then $H_{+}^{*} \subseteq H_{\geq r}^{*}$ and hence, $H_{+}^{*} \cdot H_{+}^{*} \subseteq H_{\geq 2 r}^{*}$. Thus, if $\mathcal{H}^{*} \subseteq \mathcal{A}_{d}^{*}$ is an $\imath_{0}$-adapted harmonic subspace then by (3.3) it immediately follows that

$$
\begin{array}{lll}
H^{k}=H_{\text {gen }}^{k} & \text { for } 1 \leq k \leq 2 r-1, & H^{2 r}=H_{\text {gen }}^{2 r} \oplus H^{r} \cdot H^{r}, \\
\mathcal{H}^{k}=\mathcal{H}_{\text {gen }}^{k} & \text { for } 1 \leq k \leq 2 r-1, & \mathcal{H}^{2 r} \subseteq \mathcal{H}_{\text {gen }}^{2 r} \oplus \mu\left(\mathcal{H}^{r} \odot \mathcal{H}^{r}\right),
\end{array}
$$

where $\mu$ is the multiplication map in $\mathcal{A}^{*}$. In particular, as $\mathcal{A}^{*}$ is $(r-1)$ connected, it follows that both $H_{\text {gen }}^{k}$ and $\mathcal{H}_{\text {gen }}^{k}$ vanish for $1 \leq k \leq r-1$. Moreover, most of the spaces of degree $\leq 2 r$ in (3.7) vanish because of (3.8), whence we immediately conclude

$$
\begin{aligned}
& K^{k}=\mathcal{K}^{k}=0 \quad \text { for } 1 \leq k \leq 2 r-1, \\
& K^{2 r}=\operatorname{ker}\left(\cdot: H^{r} \odot H^{r} \rightarrow H^{2 r}\right) \\
& \mathcal{K}^{2 r}=\mu\left(\mathcal{H}^{r} \odot \mathcal{H}^{r}\right) \cap d \mathcal{A}^{2 r-1} .
\end{aligned}
$$

Note that any subalgebra of $\mathcal{A}^{*}$ which contains $\mathcal{H}^{*}$ and hence $\mathcal{H}_{\text {gen }}^{*}=$ $\imath_{0}\left(H_{\text {gen }}^{*}\right)$ must also contain $\imath_{0}\left(S^{*}\left(H_{\text {gen }}^{*}\right)\right)=\mathcal{H}^{*} \oplus \mathcal{K}^{*}$, using the splitting of the bottom exact sequence in (3.7). This holds, in particular, for the small algebra $\mathcal{A}_{\text {small }}^{*}$. Comparing this with the recursive description of $\mathcal{A}_{\text {small }}^{*}$ in (3.2), this together with (3.9) yields

$$
\begin{cases}\mathcal{A}_{\text {small }}^{0}=\mathcal{H}^{0}=\mathbb{F} \cdot 1_{\mathcal{A}}, & \\ \mathcal{A}_{\text {small }}^{k}=\mathcal{H}^{k}=0, & r \leq k \leq r-1 \\ \mathcal{A}_{\text {small }}^{k}=\mathcal{H}^{k}, & \\ \mathcal{A}_{\text {small }}^{2 r-1}=\mathcal{H}^{2 r-1} \oplus d^{-} \mathcal{K}^{2 r} ; & \\ \mathcal{A}_{\text {small }}^{2 r}=\mathcal{H}^{2 r} \oplus \mathcal{K}^{2 r} \oplus d^{-} \mathcal{A}_{\text {small }}^{r, r+1} . & \end{cases}
$$

Thus, when passing to the quotient algebra $\mathcal{Q}_{\text {small }}^{k}$, we can summarize our discussion so far as follows.

Theorem 3.10. Let $\mathcal{A}^{*}$ be a $(r-1)$ connected $(r>1)$ Poincaré-DGCA of degree $n$ of Hodge type. Then $\mathcal{A}^{*}$ is equivalent to a finite dimensional non-degenerate Poincaré-DGCA $\mathcal{Q}_{\text {small }}^{*}$ of Hodge type, satisfying

- $\mathcal{Q}_{\text {small }}^{k}=\mathcal{H}^{k}$ for $0 \leq k \leq 2 r-2$ and for $n-2 r+2 \leq k \leq n$.

- if the multiplication map $\cdot: H^{r} \odot H^{r} \rightarrow H^{2 r}$ is injective, then $\mathcal{Q}_{\text {small }}^{k}=\mathcal{H}^{k}$ also for $k=2 r-1$ and $n-2 r+1$.

Proof. This is an immediate consequence of (3.10) as $\mathcal{H}^{k} \subseteq \mathcal{Q}_{\text {small }}^{k}$ and, as it is a quotient, $\operatorname{dim} \mathcal{Q}_{\text {small }}^{k} \leq \operatorname{dim} \mathcal{A}_{\text {small }}^{k}$. Also, $\operatorname{dim} \mathcal{Q}_{\text {small }}^{k}=\operatorname{dim} \mathcal{Q}_{\text {small }}^{n-k}$ as $\mathcal{Q}_{\text {small }}^{*}$ is a non-degenerate Poincaré DGCA.

The last statement follows as the injectivity of this multiplication map implies that $K^{2 r}=0$ by (3.9), whence $\mathcal{K}^{2 r}=\imath_{0}\left(K^{2 r}\right)=0$, so that $\mathcal{A}_{\text {small }}^{2 r-1}=$ $\mathcal{H}^{2 r-1}$ by (3.10). 
Corollary 3.11. Let $\mathcal{A}^{*}$ be a $(r-1)$-connected $(r>1)$ Poincaré-DGCA of Hodge type. Then $\mathcal{A}^{*}$ is equivalent to a finite dimensional non-degenerate Poincaré DGCA $\mathcal{Q}_{\text {small }}^{*}$ for which the differential d: $\mathcal{Q}_{\text {small }}^{k-1} \rightarrow \mathcal{Q}_{\text {small }}^{k}$ is possibly nonzero only for $2 r \leq k \leq n-2 r+1$. In particular

(1) If $n \leq 4 r-2$, then $\mathcal{A}^{*}$ is formal.

(2) If $n=4 r-1$, then $d: \mathcal{Q}_{\text {small }}^{k-1} \rightarrow \mathcal{Q}_{\text {small }}^{k}$ vanishes for $k \neq 2 r$.

Of course, this applies to $\mathcal{A}^{*}=\Omega^{*}(M)$ where $M$ is a closed oriented connected $n$-dimensional manifold with $b^{k}(M)=0,1 \leq k \leq r-1$.

Remark 3.12. (1) Corollary 3.11(1) for DGCAs associated with closed (orientable) manifolds has been first proved by Miller in [Miller1979] using Quillen's functor, including the statement that any closed simply connected manifold of dimension $\leq 6$ is formal. It can be also derived from Crowley-Nordström's result [CN2015, Theorem 1.3] based on their invented Bianchi-Massey tensor.

(2) If $M$ is a simply connected 7-manifold, then Corollary 3.11 implies that $M$ is almost formal in the sense of [CKT2018. That is, $\Omega^{*}(M)$ is equivalent to a DGCA $\mathcal{Q}^{*}$ whose only possibly nonvanishing differential is $d: \mathcal{Q}^{3} \rightarrow \mathcal{Q}^{4}$. This almost-formality was shown in CKT2018 for $G_{2}$-manifolds, but as our result shows, the $G_{2}$-structure is not needed.

\section{4. $A_{\infty}$-QUasi-isomorphism type of highly Connected Poincaré DGCAS OF HODGE TYPE}

In this section, using homotopy transfer theorem and related technique, we prove that a $(r-1)$ connected Poincare DGCA $\mathcal{A}^{*}$ of Hodge type of dimension $n \leq 5 r-3$ is $A_{\infty}$-quasi-isomorphic to an $A_{3}$-algebra (Theorem 4.1), that the only obstruction to the formality of $\mathcal{A}^{*}$ is the cohomology class $\left[\mu_{3}\right] \in \operatorname{Hoch}^{2}\left(H^{*}\left(\mathcal{A}^{*}\right), H^{*}\left(\mathcal{A}^{*}\right)\right)$, where $\mu_{3}$ is the class of the ternary multiplication in the $A_{3}$-algebra (Theorem 4.6), and that the cohomology class $\left[\mu_{3}\right]$ and the DGCA isomorphism class of $H^{*}\left(\mathcal{A}^{*}\right)$ determine the $A_{\infty}$-quasiisomorphism class of $\mathcal{A}^{*}$ (Theorem 4.7). Then we show the relation between $\mu_{3}$ and the Massey product (Remark 4.8, Corollary 4.13) and compare our result with a closely related result by Crowley-Nordström (Remark 4.12).

Theorem 4.1. Let $\mathcal{A}^{*}$ be a $(r-1)$-connected $(r>1)$ Poincaré DGCA of degree $n$, endowed with a Hodge-type decomposition whose harmonic subspace $\mathcal{H}^{*}$ is adapted to a generating subspace $H_{\text {gen }}^{*}$. If $n \leq 5 r-3$ then $\mathcal{H}^{*}$ carries an $A_{3}$-algebra structure $\mathbb{1}^{1}$ (with trivial differential) making it $A_{\infty}$-quasiisomorphic to $\mathcal{A}^{*}$. Moreover, if $n \leq 4 r-2$ then the ternary multiplication of this $A_{3}$-algebra vanishes, so in particular $\mathcal{A}^{*}$ is formal.

Proof. Let us therefore focus on the case $4 r-1 \leq n \leq 5 r-3$. As we know that the smallest $\mathcal{H}^{*}$-quotient $\mathcal{Q}_{\text {small }}^{*}$ of $\mathcal{A}^{*}$ is equivalent to $\mathcal{A}^{*}$ by Corollary

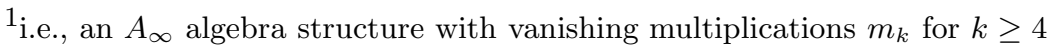


3.11, we only need to prove that $\mathcal{H}^{*}$ carries an $A_{3}$-algebra structure with trivial differential making it quasi-isomorphic to $\mathcal{Q}_{\text {small }}^{*}$. We can show this by means of the homotopy transfer theorem Kadeishvili1980, Merkulov1999.

By Poincaré duality and equation (3.10) $\mathcal{Q}_{\text {small }}^{*}$ is given by

$$
\left\{\begin{array}{lrl}
\mathcal{Q}_{\text {small }}^{0} & =\mathcal{H}^{0} \cong \mathbb{F} & \\
\mathcal{Q}_{\text {small }}^{k} & =\mathcal{H}^{k}=0, & 1 \leq k \leq r-1 \\
\mathcal{Q}_{\text {small }}^{k} & =\mathcal{H}^{k}, & r \leq k \leq 2 r-2 \\
\mathcal{Q}_{\text {small }}^{k} & =\mathcal{H}^{k} \oplus \mathcal{L}^{k}, & 2 r-1 \leq k \leq n-2 r+1 \\
\mathcal{Q}_{\text {small }}^{k}=\mathcal{H}^{k}, & n-2 r+2 \leq k \leq n-r \\
\mathcal{Q}_{\text {small }}^{k}=\mathcal{H}^{k}=0, & n-r+1 \leq k \leq n-1 \\
\mathcal{Q}_{\text {small }}^{n}=\mathcal{H}^{n} \cong \mathbb{F} . &
\end{array}\right.
$$

where $\mathcal{L}^{k}=d d^{-} \mathcal{Q}_{\text {small }}^{k} \oplus d^{-} d \mathcal{Q}_{\text {small }}^{k}$ for every $k$ and, in particular

$$
\mathcal{L}^{2 k-1}=d^{-} \mu\left(\mathcal{H}^{k} \odot \mathcal{H}^{k}\right) .
$$

Notice that $d^{-}$identically vanishes on $\mathcal{Q}_{\text {small }}^{k}$ for $k \leq 2 r-1$ and for $k \geq$ $n-2 r+2$. By the homotopy transfer theorem, $\mathcal{H}^{*}$ carries an $A_{\infty}$-algebra structure making it $A_{\infty}$-quasi-isomorphic to $\mathcal{Q}_{\text {small }}^{*}$. To prove the statement in the theorem we therefore only need to show that this $A_{\infty}$-algebra structure is actually an $A_{3^{-}}$algebra structure, i.e., that all the multiplications $m_{k}$ vanish for $k \geq 4$. One has a convenient tree summation formula to express the higher multiplications $m_{k}$ obtained by homotopy transfer, see [KS2001]. Namely, $m_{k}$ can be expressed as a sum over rooted trivalent trees with $k$ leaves. Each tail edge of such a tree is decorated by inclusion $j: \mathcal{H}^{*} \hookrightarrow$ $\mathcal{Q}_{\text {small }}^{*}$, each internal edge is decorated by the operator $d^{-}: \mathcal{Q}_{\text {small }}^{*} \rightarrow \mathcal{Q}_{\text {small }}^{*-1}$ and the root edge is decorated by the operator $\pi_{\mathcal{H}^{*}}: \mathcal{Q}_{\text {small }}^{*} \rightarrow \mathcal{H}^{*}$; every internal vertex is decorated by the multiplication $\mu$ in $\mathcal{Q}_{\text {small }}^{*}$.

In order to get a nonzero operation, we can not have a subgraph of the form

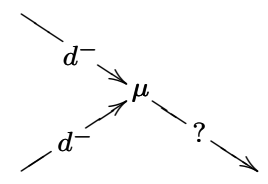

where ? can be either $d^{-}$or $\pi_{\mathcal{H}^{*}}$. Namely, in order to get a possibly nonzero contribution from this graph, its homogeneous entries $a_{k_{1}}$ and $b_{k_{2}}$ should be in $\mathcal{Q}_{\text {small }}^{k_{1}}$ and $\mathcal{Q}_{\text {small }}^{k_{2}}$ with $k_{1}, k_{2} \geq 2 r$. The term $d^{-} a_{k_{1}} \cdot d^{-} b_{k_{2}}$ will then be in $\mathcal{Q}_{\text {small }}^{k}$ with $k \geq 4 r-2$, where $k=k_{1}+k_{2}-2$. As $n \leq 5 r-3$ we have $4 r-2 \geq n-r+1$, and so $k \geq n-r+1$. Therefore, $d^{-} a_{k_{1}} \cdot d^{-} b_{k_{2}}$ is surely zero unless $k_{1}+k_{2}-2=n$. For $k_{1}+k_{2}-2=n$ we can compute

$$
\int d^{-} a_{k_{1}} \cdot d^{-} b_{k_{2}}=\left\langle d^{-} a_{k_{1}}, d^{-} b_{k_{2}}\right\rangle=0
$$

by the orthogonality relation (2.11). As $\int: \mathcal{Q}_{\text {small }}^{n} \rightarrow \mathbb{R}$ is an isomorphism, this gives $d^{-} a_{k_{1}} \cdot d^{-} b_{k_{2}}=0$. 
Also, we can not have a subgraph of the form

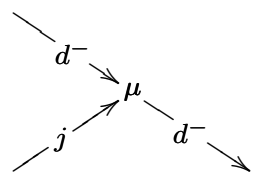

Namely, if its homogeneous entries are $a_{k_{1}}$ and $b_{k_{2}}$ (from top to bottom), then we need to have $k_{1} \geq 2 r$ and $k_{1}+k_{2}-1 \leq n-2 r+1$ in order to have a possibly nonzero contribution. The two inequalities together give $k_{2} \leq n-4 r+2 \leq r-1$. So the only possibility for having a nonzero contribution is $k_{2}=0$. As $\mathcal{Q}_{\text {small }}^{0}=\mathbb{F}$ acting as scalars on $\mathcal{Q}_{\text {small }}^{*}$ via the multiplication $\mu$, we have

$$
d^{-}\left(d^{-} a_{k_{1}} \cdot b_{0}\right)=b_{0}\left(d^{-}\right)^{2} a_{k_{1}}=0 .
$$

In conclusion, the only two graphs actually appearing in the tree summation formula are (absorbing the permutations of the tree branches in the graded commutativity of the multiplication) are

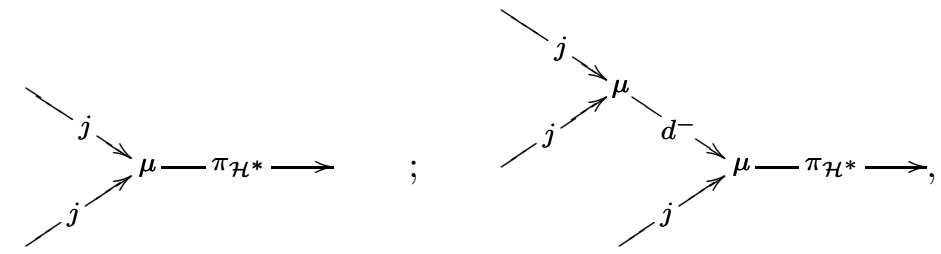

defining the multiplications $m_{2}: \mathcal{H}^{k_{1}} \otimes \mathcal{H}^{k_{2}} \rightarrow \mathcal{H}^{k_{1}+k_{2}}$ and $m_{3}: \mathcal{H}^{k_{1}} \otimes \mathcal{H}^{k_{2}} \otimes$ $\mathcal{H}^{k_{3}} \rightarrow \mathcal{H}^{k_{1}+k_{2}+k_{2}-1}$, respectively.

If $n \leq 4 r-2$, we can verbatim repeat the above argument, but now all the $\mathcal{L}^{k} ; \mathrm{s}$ are zero so also the multiplication $m_{3}$ vanishes. The $A_{3}$-algebra structure on $\mathcal{H}^{*}$ therefore becomes a DGCA structure with trivial differential, and we recover the formality of $\mathcal{A}^{*}$ in this case (Corollary 3.11).

Remark 4.2. The multiplications $m_{2}$ and $m_{3}$ of the $A_{3}$ algebra structure on $\mathcal{H}^{*}$ described in the proof of Theorem 4.1 are explicitly given by

$$
\begin{aligned}
m_{2}(\alpha, \beta) & =\pi_{\mathcal{H}^{*}}(\alpha \cdot \beta) \\
m_{3}(\alpha, \beta, \gamma) & =\pi_{\mathcal{H}^{*}}\left(d^{-}(\alpha \cdot \beta) \cdot \gamma-(-1)^{\operatorname{deg} \alpha} \alpha \cdot d^{-}(\beta \cdot \gamma)\right)
\end{aligned}
$$

see [Merkulov1999, Theorem 3.4]. Moreover, as the pairing $\langle-,-\rangle$ on $\mathcal{H}^{*}$ is nondegenerate, the ternary multiplication $m_{3}$ is completely encoded in the tensor

$$
\tau(\alpha, \beta, \gamma, \delta)=\left\langle m_{3}(\alpha, \beta, \gamma), \delta\right\rangle
$$

Remark 4.3. It is immediate to see that

$$
m_{3}(\alpha, \beta, \gamma)=0 \quad \text { if } \quad \min \{\operatorname{deg} \alpha, \operatorname{deg} \beta, \operatorname{deg} \gamma\}=0 .
$$

Indeed, the operator $d^{-}$vanishes on $\mathcal{H}^{*}$, the projection $\pi_{\mathcal{H}^{*}}$ vanishes on the image of $d^{-}$and the elements in $\mathcal{H}^{0} \subseteq \mathcal{A}^{0}$ act as scalars via the multiplication in $\mathcal{A}^{*}$. From this one sees that the expression for $m_{3}$ given in Remark 4.2 identically vanishes when one of the arguments of $m_{3}$ has degree zero. 
Corollary 4.4. Let $\mathcal{A}^{*}$ a $(r-1)$-connected $(r>1)$ Poincaré DGCA of degree $n$ of Hodge type with an adapted choice $\imath: H^{*}\left(\mathcal{A}^{*}\right) \hookrightarrow \mathcal{A}_{d}^{*}$ of harmonic forms. If $n \leq 5 r-3$, then $H^{*}\left(\mathcal{A}^{*}\right)$ carries an $A_{3}$-algebra structure with zero differential, whose binary multiplication is the the multiplication induced by $\mathcal{A}^{*}$, while the ternary multiplication is

$$
\mu_{3}([\alpha],[\beta],[\gamma])=\left[m_{3}(\imath[\alpha], \imath[\beta], \imath[\gamma])\right]
$$

making it $A_{\infty}$-quasi-isomorphic to $\mathcal{A}^{*}$. Moreover, if $n \leq 4 r-2$ then $\mu_{3}$ vanishes.

Proof. The isomorphism $\imath: H^{*}\left(\mathcal{A}^{*}\right) \stackrel{\sim}{\rightarrow} \mathcal{H}^{*}$ with inverse $\pi_{\mathcal{H}^{*}}: \mathcal{H}^{*} \stackrel{\sim}{\rightarrow} H^{*}\left(\mathcal{A}^{*}\right)$ transfers the $A_{3}$-algebra structure on $\mathcal{H}^{*}$ from Theorem 4.1 to an $A_{3}$-algebra structure with the same properties on $H^{*}\left(\mathcal{A}^{*}\right)$. So the only thing we need to prove is the identity

$$
\left[m_{2}(\imath[\alpha], \imath[\beta])\right]=[\alpha] \cdot[\beta] .
$$

This is straightforward, as

$$
[\alpha] \cdot[\beta]=[(\imath[\alpha]) \cdot(\imath[\beta])]=\left[\pi_{\mathcal{H}^{*}}((\imath[\alpha]) \cdot(\imath[\beta]))\right]=\left[m_{2}(\imath[\alpha], \imath[\beta])\right] .
$$

Lemma 4.5. The degree - 1 trilinear map $\left.\mu_{3}: H^{k_{1}}\left(\mathcal{A}^{*}\right) \otimes H^{k_{2}}\left(\mathcal{A}^{*}\right)\right) \otimes H^{k_{3}}\left(\mathcal{A}^{*}\right) \rightarrow$ $H^{k_{1}+k_{2}+k_{3}-1}\left(\mathcal{A}^{*}\right)$ from Corollary 4.4 is a cocycle in the Hochschild complex of the DGCA $H^{*}\left(\mathcal{A}^{*}\right)$, and so it defines a Hochschild cohomology class $\left[\mu_{3}\right] \in \operatorname{Hoch}^{3,-1}\left(H^{*}\left(\mathcal{A}^{*}\right), H^{*}\left(\mathcal{A}^{*}\right)\right) \subseteq \operatorname{Hoch}^{2}\left(H^{*}\left(\mathcal{A}^{*}\right), H^{*}\left(\mathcal{A}^{*}\right)\right)$.

Proof. Immediate from Corollary 4.4, see, e.g., [Lunts2007] for details.

Theorem 4.6. Let $\mathcal{A}^{*}$ a $(r-1)$-connected $(r>1)$ Poincaré DGCA of degree $n$ of Hodge type with $n \leq 5 r-3$. The Hochschild cohomology class $\left[\mu_{3}\right]$ is independent of the choice of adapted harmonic forms and of associated Hodge decomposition, and so it defines a distinguished element

$$
\left[\mu_{3}^{\mathcal{A}^{*}}\right] \in \operatorname{Hoch}^{2}\left(H^{*}\left(\mathcal{A}^{*}\right), H^{*}\left(\mathcal{A}^{*}\right)\right)
$$

depending only on the Poincaré DGCA $\mathcal{A}^{*}$. Moreover the class $\left[\mu_{3}^{\mathcal{A}^{*}}\right]$ is the only obstruction to the formality of $\mathcal{A}^{*}$.

Proof. It follows by a general result by Kadeishvili Kadeishvili1988 and Kaledin Kaledin2005 that the only obstruction to the formality of an $A_{3}$-algebra with trivial differential is the Hochschild cohomology class of the trilinear multiplication, see [Lunts2007, Corollary 5.7]. Therefore, as $\left(H^{*}\left(\mathcal{A}^{*}\right), 0, \cdot, \mu_{3}\right)$ and $\left(\mathcal{A}^{*}, d, \cdot\right)$ are $A_{\infty}$-quasi-isomorphic, $\left[\mu_{3}\right]$ is the only obstruction to the formality of $\mathcal{A}^{*}$. To conclude, we need to show that the class $\left[\mu_{3}\right]$ is actually independent of the choice of adapted harmonic forms and of associated Hodge decomposition. To see this, let $\tilde{\iota}$ and $\tilde{d}^{-}$be the operators occurring in a different choice, and let $\tilde{\mu}_{3}$ be the associated the trilinear multiplication. Then we will have $A_{\infty}$-quasi-isomorphism

$$
\imath_{\infty}:\left(H^{*}\left(\mathcal{A}^{*}\right), 0, \cdot \mu_{3}\right) \rightarrow\left(\mathcal{A}^{*}, d, \cdot\right) ; \quad \tilde{\imath}_{\infty}:\left(H^{*}\left(\mathcal{A}^{*}\right), 0, \cdot, \tilde{\mu}_{3}\right) \rightarrow\left(\mathcal{A}^{*}, d, \cdot\right)
$$


extending the linear morphisms $\imath$ and $\tilde{\imath}$, respectively. By considering an $A_{\infty^{-}}$ up to homotopy inverse quasi-isomorphism $\tilde{\pi}_{\infty}:\left(\mathcal{A}^{*}, d, \cdot\right) \rightarrow\left(H^{*}\left(\mathcal{A}^{*}\right), 0, \cdot, \tilde{\mu}_{3}\right)$ we therefore get a homotopy commutative triangle of $A_{\infty}$-quasiisomorphisms

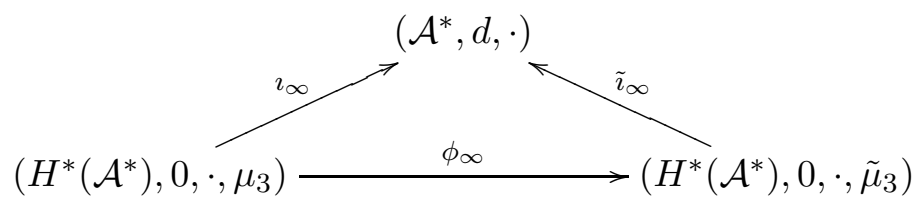

for some $A_{\infty}$-quasi-isomorphism $\phi_{\infty}$. Passing to cohomology we get the commutative diagram

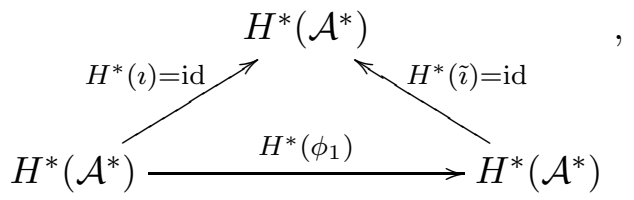

where $\phi_{1}$ is the linear component of $\phi_{\infty}$. But then $\phi_{1}=\mathrm{id}: H^{*}\left(\mathcal{A}^{*}\right) \rightarrow$ $H^{*}\left(\mathcal{A}^{*}\right)$, and so

$$
\phi_{\infty}:\left(H^{*}\left(\mathcal{A}^{*}\right), 0, \cdot, \mu_{3}\right) \rightarrow\left(H^{*}\left(\mathcal{A}^{*}\right), 0, \cdot \tilde{\mu}_{3}\right)
$$

is an $A_{\infty}$-quasi-isomorphism whose linear component is the identity. Spelling out the trilinear component in the definition of $A_{\infty}$-morphism, i.e.,

$$
\sum_{\substack{r+s+t=3 \\ r, t \geq 0, s \geq 1}}(-1)^{r s+t} \phi_{r+1+t}\left(\mathrm{id}^{\otimes r} \otimes \mu_{s} \otimes \mathrm{id}^{\otimes t}\right)=\sum_{\substack{j \in\{1,2,3\} \\ i_{1}+\cdots+i_{j}=3 \\ i_{1}, \ldots, i_{j} \geq 1}}(-1)^{u} \tilde{\mu}_{j}\left(\phi_{i_{1}} \otimes \cdots \otimes \phi_{i_{j}}\right),
$$

where $u=\sum_{k=1}^{j-1}(j-k)\left(i_{k}-1\right)$, and using the fact $\phi_{1}=\mathrm{id}$, and that on both sides the differentials $\mu_{1}$ and $\tilde{\mu}_{1}$ are zero and the binary multiplications $\mu_{2}$ and $\tilde{\mu}_{2}$ are the multiplication $m$ in $H^{*}\left(\mathcal{A}^{*}\right)$, one finds

$$
\mu_{3}-\phi_{2}(m \otimes \mathrm{id})+\phi_{2}(\mathrm{id} \otimes m)=m\left(\mathrm{id} \otimes \phi_{2}\right)-m\left(\phi_{2} \otimes \mathrm{id}\right)+\tilde{\mu}_{3},
$$

i.e., precisely,

and so $\left[\tilde{\mu}_{3}\right]=\left[\mu_{3}\right]$.

$$
\tilde{\mu}_{3}=\mu_{3}+d_{\text {Hoch }} \phi_{2},
$$

The same argument used in the proof of Theorem 4.6 shows that the Hochschild class $\left[\mu_{3}^{\mathcal{A}^{*}}\right]$ detects the $A_{\infty}$-quasi-isomorphism class of an highly connected Poincaré DGCA $\mathcal{A}^{*}$. The precise statement is the following.

Theorem 4.7. Let $\mathcal{A}^{*} a(r-1)$-connected $(r>1)$ Poincaré DGCA of Hodge type of degree $n$ with $n \leq 5 r-3$. The $A_{\infty}$-quasi-isomorphism class of $\mathcal{A}^{*}$ is completely encoded into the DGCA $\left(H^{*}\left(\mathcal{A}^{*}\right), \cdot\right)$ and in the Hochschild class $\left[\mu_{3}^{\mathcal{A}^{*}}\right] \in \operatorname{Hoch}^{2}\left(H^{*}\left(\mathcal{A}^{*}\right), H^{*}\left(\mathcal{A}^{*}\right)\right)$.

Proof. We need to show that two $(r-1)$-connected Poincaré DGCA's of Hodge type of degree at most $5 r-3, \mathcal{A}_{1}^{*}$ and $\mathcal{A}_{2}^{*}$ are $A_{\infty}$-quasi-isomorphic if and only if there exists a DGCA isomorphism $\phi: H^{*}\left(\mathcal{A}_{1}^{*}\right) \rightarrow H^{*}\left(\mathcal{A}_{2}^{*}\right)$ such 
that $\left[\phi^{-1} \circ\left(\mu_{3}^{\mathcal{A}_{2}^{*}}(\phi \otimes \phi \otimes \phi)\right)\right]=\left[\mu_{3}^{\mathcal{A}_{1}^{*}}\right]$. In one direction, if $\phi_{\infty}: \mathcal{A}_{1}^{*} \rightarrow \mathcal{A}_{2}^{*}$ is an $A_{\infty}$-quasi-isomorphism, then the morphism $\phi: H^{*}\left(\mathcal{A}_{1}^{*}\right) \rightarrow H^{*}\left(\mathcal{A}_{2}^{*}\right)$ induced by the linear part $\phi_{1}$ of $\phi_{\infty}$ is an isomorphism of DGCA's (with trivial differential). The fact that $\phi_{\infty}$ is an $A_{\infty}$-morphism then gives, in particular, the identity

$\phi\left(\mu_{3}^{\mathcal{A}_{1}^{*}}\right)=\mu_{3}^{\mathcal{A}_{2}^{*}}(\phi \otimes \phi \otimes \phi)+\phi_{2}\left(\mu_{2}^{\mathcal{A}_{1}^{*}} \otimes \mathrm{id}\right)-\phi_{2}\left(\mathrm{id} \otimes \mu_{2}^{\mathcal{A}_{1}^{*}}\right)+\mu_{2}^{\mathcal{A}_{2}^{*}}\left(\phi \otimes \phi_{2}\right)-\mu_{2}^{\mathcal{A}_{2}^{*}}\left(\phi_{2} \otimes \phi\right)$, where $\mu_{2}^{\mathcal{A}_{i}^{*}}$ is the multiplication in $H^{*}\left(\mathcal{A}_{i}^{*}\right)$. By applying $\phi^{-1}$ on both sides and using that $\phi$ is a DGCA isomorphism, we find

$$
\begin{aligned}
\mu_{3}^{\mathcal{A}_{1}^{*}}=\phi^{-1}\left(\mu_{3}^{\mathcal{A}_{2}^{*}}(\phi \otimes \phi \otimes \phi)\right) & +\left(\phi^{-1} \circ \phi_{2}\right)\left(\mu_{2}^{\mathcal{A}_{1}^{*}} \otimes \mathrm{id}\right)-\left(\phi^{-1} \circ \phi_{2}\right)\left(\mathrm{id} \otimes \mu_{2}^{\mathcal{A}_{1}^{*}}\right) \\
& +\mu_{2}^{\mathcal{A}_{1}^{*}}\left(\mathrm{id} \otimes\left(\phi^{-1} \circ \phi_{2}\right)\right)-\mu_{2}^{\mathcal{A}_{1}^{*}}\left(\left(\phi^{-1} \circ \phi_{2}\right) \otimes \mathrm{id}\right),
\end{aligned}
$$

and so $\left[\mu_{3}^{\mathcal{A}_{1}^{*}}\right]=\left[\phi^{-1} \circ\left(\mu_{3}^{\mathcal{A}_{2}^{*}}(\phi \otimes \phi \otimes \phi)\right)\right]$. Vice versa, assume we are given a DGCA isomorphism $\phi: H^{*}\left(\mathcal{A}_{1}^{*}\right) \rightarrow H^{*}\left(\mathcal{A}_{2}^{*}\right)$ such that $\left[\phi^{-1} \circ\left(\mu_{3}^{\mathcal{A}_{2}^{*}}(\phi \otimes \phi \otimes\right.\right.$ $\phi))]=\left[\mu_{3}^{\mathcal{A}_{1}^{*}}\right]$. Then, by definition of Hochschild cohomology, there exists a bilinear morphism

$$
\psi: H^{*}\left(\mathcal{A}_{1}^{*}\right) \otimes H^{*}\left(\mathcal{A}_{1}^{*}\right) \rightarrow H^{*-1}\left(\mathcal{A}_{1}^{*}\right)
$$

such that

$$
\begin{aligned}
\mu_{3}^{\mathcal{A}_{1}^{*}}=\phi^{-1}\left(\mu_{3}^{\mathcal{A}_{2}^{*}}(\phi \otimes \phi \otimes \phi)\right) & +\psi\left(\mu_{2}^{\mathcal{A}_{1}^{*}} \otimes \mathrm{id}\right)-\psi\left(\mathrm{id} \otimes \mu_{2}^{\mathcal{A}_{1}^{*}}\right) \\
& +\mu_{2}^{\mathcal{A}_{1}^{*}}(\mathrm{id} \otimes \psi)-\mu_{2}^{\mathcal{A}_{1}^{*}}(\psi \otimes \mathrm{id}) .
\end{aligned}
$$

Setting $\phi_{1}=\phi$ and $\phi_{2}=\phi \circ \psi$, the above identity precisely says that

$$
\left(\phi_{1}, \phi_{2}\right):\left(H^{*}\left(\mathcal{A}_{1}^{*}\right), \mu_{2}^{\mathcal{A}_{1}^{*}}, \mu_{3}^{\mathcal{A}_{1}^{*}}\right) \rightarrow\left(H^{*}\left(\mathcal{A}_{2}^{*}\right), \mu_{2}^{\mathcal{A}_{2}^{*}}, \mu_{3}^{\mathcal{A}_{3}^{*}}\right)
$$

is an $A_{\infty}$-isomorphism of $A_{3}$-algebras with trivial differential. As we have $A_{\infty}$-quasi-isomorphisms $\mathcal{A}_{1}^{*} \cong\left(H^{*}\left(\mathcal{A}_{1}^{*}\right), \mu_{2}^{\mathcal{A}_{1}^{*}}, \mu_{3}^{\mathcal{A}_{1}^{*}}\right)$ and $\mathcal{A}_{2}^{*} \cong\left(H^{*}\left(\mathcal{A}_{2}^{*}\right), \mu_{2}^{\mathcal{A}_{2}^{*}}, \mu_{3}^{\mathcal{A}_{2}^{*}}\right)$, this concludes the proof.

Remark 4.8 (Massey products). The ternary multiplication $\mu_{3}([\alpha],[\beta],[\gamma])$ is particularly interesting when both the products $[\alpha] \cdot[\beta]$ and $[\beta] \cdot[\gamma]$ vanish. Indeed, in this case we have

$$
\left\{\begin{array}{l}
\iota[\alpha] \cdot \iota[\beta]=d \eta \\
\iota[\alpha] \cdot \iota[\beta]=d \theta
\end{array}\right.
$$

for suitable elements $\eta, \theta \in d^{-} \mathcal{A}^{*}$ and so

$$
\begin{aligned}
\mu_{3}([\alpha],[\beta],[\gamma]) & =\left[\pi_{\mathcal{H}^{*}}\left(\eta \cdot \iota[\gamma]-(-1)^{\operatorname{deg} \alpha} \iota[\alpha] \cdot \theta\right)\right] \\
& =\left[\eta \cdot \iota[\gamma]-(-1)^{\operatorname{deg} \alpha} \iota[\alpha] \cdot \theta\right],
\end{aligned}
$$

as $\eta \cdot \iota[\gamma]-(-1)^{\operatorname{deg} \alpha} \iota[\alpha] \cdot \theta$ is $d$-closed. This equation precisely states that $\mu_{3}([\alpha],[\beta],[\gamma])$ is a representative for the triple Massey product of $[\alpha],[\beta]$ and $[\gamma]$. In particular, we see that if $\mu_{3}$ vanishes, then all triple Massey products vanish. One should however be warned that the converse is not true: there 
are examples of non-formal algebras with vanishing Massey products. See BMFM2018, Valette2012 for a detailed description of the relation between the $A_{\infty}$-algebra structure on $H^{*}\left(\mathcal{A}^{*}\right)$ obtained by homotopy transfer from the DGCA structure on a DGCA $\mathcal{A}^{*}$ and the (triple and higher) Massey products on $H^{*}\left(\mathcal{A}^{*}\right)$.

Remark 4.9. Although an $A_{3}$-algebra can generally have nontrivial quadruple Massey products (see [BMFM2018, Theorem D]), simple degree considerations show that if $\mathcal{A}^{*}$ is a $(r-1)$-connected $(r>1)$ Poincaré DGCA of degree $n \leq 5 r-3$ then all of the $l$ th order Massey products are trivial for $l \geq 4$. We show this in Appendix A.

Lemma 4.10. The degree - 1 trilinear map $\mu_{3}: H^{k_{1}}\left(\mathcal{A}^{*}\right) \otimes H^{k_{2}}\left(\mathcal{A}^{*}\right) \otimes H^{k_{3}}\left(\mathcal{A}^{*}\right) \rightarrow$ $H^{k_{1}+k_{2}+k_{3}-1}\left(\mathcal{A}^{*}\right)$ from Corollary 4.4 is a 2-cocycle in the Harrison subcomplex $\operatorname{Harr}^{*}\left(H^{*}\left(\mathcal{A}^{*}\right), H^{*}\left(\mathcal{A}^{*}\right)\right) \subseteq \operatorname{Hoch}^{*}\left(H^{*}\left(\mathcal{A}^{*}\right), H^{*}\left(\mathcal{A}^{*}\right)\right)$. In particular it defines an element in $\operatorname{Harr}^{3,-1}\left(H^{*}\left(\mathcal{A}^{*}\right), H^{*}\left(\mathcal{A}^{*}\right)\right) \subseteq \operatorname{Harr}^{2}\left(H^{*}\left(\mathcal{A}^{*}\right), H^{*}\left(\mathcal{A}^{*}\right)\right)$.

Proof. By definition of the Harrison complex [Harrison1962, we have to show that

$$
\mu_{3}([\alpha],[\beta],[\gamma])-(-1)^{\overline{[\alpha] \cdot[\beta]}} \mu_{3}([\beta],[\alpha],[\gamma])+\mu_{3}([\beta],[\gamma],[\alpha])=0
$$

for any $[\alpha],[\beta],[\gamma]$ in $H^{*}\left(\mathcal{A}^{*}\right)$, where we have written $\overline{[x]}$ for the degree of the homogeneous element $[x] \in H^{*}\left(\mathcal{A}^{*}\right)$. We compute

$$
\begin{aligned}
& \mu_{3}([\alpha],[\beta],[\gamma])-(-1)^{\overline{[\alpha]} \cdot \overline{[\beta]}} \mu_{3}([\beta],[\alpha],[\gamma])+(-1)^{\overline{[\alpha]} \cdot(\overline{[\beta]}+\overline{[\gamma]})} \mu_{3}([\beta],[\gamma],[\alpha])= \\
& =\left[m_{3}(\imath[\alpha], \imath[\beta], \imath[\gamma])\right]-(-1)^{\overline{[\alpha] \cdot[\beta]}}\left[m_{3}(\imath[\beta], \imath[\alpha], \imath[\gamma])\right]+(-1)^{\overline{\alpha \alpha]} \cdot(\overline{[\beta]}+\overline{[\gamma]})}\left[m_{3}(\imath[\beta], \imath[\gamma], \imath[\alpha])\right] \\
& =\pi_{\mathcal{H}^{*}}\left(d^{-}(\imath[\alpha] \cdot \imath[\beta]) \cdot \imath[\gamma]-(-1)^{\overline{[\alpha]}} \imath[\alpha] \cdot d^{-}(\imath[\beta] \cdot \imath[\gamma])\right) \\
& +\pi_{\mathcal{H}^{*}}\left(-(-1)^{\overline{[\alpha]} \cdot \overline{[\beta]}} d^{-}(\imath[\beta] \cdot \imath[\alpha]) \cdot \imath[\gamma]+(-1)^{(\overline{[\alpha]}+1)[\overline{[\beta]}} \imath[\beta] \cdot d^{-}(\imath[\alpha] \cdot \imath[\gamma])\right) \\
& +\pi_{\mathcal{H}^{*}}\left((-1)^{\overline{[\alpha]} \cdot(\overline{[\beta]}+\overline{[\gamma]})} d^{-}(\imath[\beta] \cdot \imath[\gamma]) \cdot \imath[\alpha]-(-1)^{(\overline{[\alpha]}+1) \overline{[\beta]}+\overline{[\alpha]} \cdot \overline{[\gamma]}} \imath[\beta] \cdot d^{-}(\imath[\gamma] \cdot \imath[\alpha])\right) \\
& =0 \text {, }
\end{aligned}
$$

as the multiplication in $\mathcal{A}^{*}$ is graded commutative.

Since in characteristic zero the Harrison cohomology of a DGCA injects into the Hochschild cohomology [Barr1968, we have this immediate corollary of Theorems 4.6 and 4.7.

Corollary 4.11. Let $\mathcal{A}^{*} a(r-1)$-connected $(r>1)$ Poincaré DGCA of

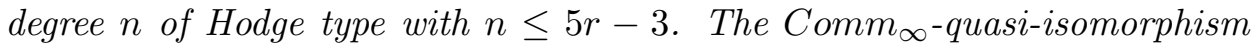
class of $\mathcal{A}^{*}$ is completely encoded into the DGCA $\left(H^{*}\left(\mathcal{A}^{*}\right), \cdot\right)$ and in the Harrison cohomology class $\left[\mu_{3}^{\mathcal{A}^{*}}\right] \in \operatorname{Harr}^{2}\left(H^{*}\left(\mathcal{A}^{*}\right), H^{*}\left(\mathcal{A}^{*}\right)\right)$. In particular, the Harrison class $\left[\mu_{3}^{\mathcal{A}^{*}}\right]$ is the only obstruction to the formality of $\mathcal{A}^{*}$.

Remark 4.12 (The Bianchi-Massey tensor). Via the spectral sequence relating Harrison to André-Quillen cohomology [Barr1968, Loday1992, the Harrison cohomology class $\left[\mu_{3}^{\mathcal{A}^{*}}\right] \in \operatorname{Harr}^{3,-1}\left(H^{*}\left(\mathcal{A}^{*}\right), H^{*}\left(\mathcal{A}^{*}\right)\right)$ is equally represented by an element $\beta^{\mathcal{A}^{*}} \in \operatorname{Hom}^{-1}\left(S^{2}\left(S H^{*}\left(\mathcal{A}^{*}\right)\right), H^{*}\left(\mathcal{A}^{*}\right)\right)$. This latter 
element is the Bianchi-Massey tensor introduced in CN2015 and therein identified as the only obstruction to the formality of a $r$-connected $(r>1)$ Poincaré DGCA $\mathcal{A}^{*}$ of degree $n$ with $n \leq 5 r-3$, as well as a complete invariant of the quasi-isomorphism class of such an $\mathcal{A}^{*}$, together with the cohomology algebra $H^{*}\left(\mathcal{A}^{*}\right)$. In other words, Corollary 4.11 precisely reproduces the main results from CN2015, and could be obtained from them by translating the Bianchi-Massey tensor into the corresponding Harrison cohomology class. It should however be remarked that our derivation of Corollary 4.11 is independent of CN2015 and can therefore be read as a confirmation of their results.

From a computational point of view, it seems that the vanishing of the Bianchi-Massey tensor is easier to be checked than the vanishing of the corresponding Harrison cohomology class, so that the CN2015 approach appears to be more suitable in investigating formality of a given highly connected manifold. The approach via homotopy transfer and Hochschild/Harrison cohomology, on the other hand, appears to be more fitting for the investigation of Massey products. We give an example in Corollaries 4.13,4.14] below.

Corollary 4.13. Let $\mathcal{A}^{*}$ be a simply connected Poincaré DGCA of degree $n$ of Hodge type.

(1) If $n \leq 6$, then the Massey product of any Massey tripld vanishes.

(2) If $n=7$, then the Massey product vanishes for every Massey triple of degree $\neq(2,2,2)$.

(3) If $n=8$, then the Massey product vanishes for every Massey triple of degree $\notin\{(2,2,2),(2,2,3),(2,3,2),(3,2,2)\}$.

Proof. The statement is trivial if $n \leq 6$ as in this case $\mathcal{A}^{*}$ is formal by Corollary 3.11. When the degree of $\mathcal{A}^{*}$ is 7 , we show that $\mu_{3}([\alpha],[\beta],[\gamma])$ is possibly nonzero only when $\operatorname{deg}([\alpha],[\beta],[\gamma])=(2,2,2)$. The conclusion then follows by Remark 4.8. By Corollary 4.4, we are reduced to show that $\mu_{3}(\alpha, \beta, \gamma)=0$ for any three harmonic elements $\alpha, \beta, \gamma$ with $\operatorname{deg}(\alpha, \beta, \gamma) \neq(2,2,2)$. By (4.1) we see that for a degree-7 algebra $\mathcal{A}^{*}$ the operator $d^{-}: \mathcal{Q}_{\text {small }}^{k} \rightarrow \mathcal{Q}_{\text {small }}^{k-1}$ is identically zero unless $k=4$. From the explicit formula for $m_{3}$ given in Remark 4.2, we therefore see that $\mu_{3}(\alpha, \beta, \gamma)=0$ unless $\operatorname{deg} \alpha+\operatorname{deg} \beta=4$ or $\operatorname{deg} \beta+\operatorname{deg} \gamma=4$. As $\mathcal{A}^{*}$ is simply connected, and by Remark 4.3, $\mu_{3}(\alpha, \beta, \gamma)$ is zero if one of the elements $\alpha, \beta, \gamma$ has degree 0 or 1 . So the only possible nonvanishing triple products come form triples with degrees $(2,2, k)$ or $(k, 2,2)$. If $(\alpha, \beta, \gamma)$ is such a triple, then $\mu_{3}(\alpha, \beta, \gamma) \in \mathcal{H}^{k+3}$. So having a possibly nonzero result restricts to the two possibilities $k=2$ or $k=4$. To rule out the $k=4$ possibility, notice that if $\operatorname{deg}(\alpha, \beta, \gamma)=(2,2,4)$, then

$$
\int m_{3}(\alpha, \beta, \gamma)=\int \pi_{\mathcal{H}^{*}}\left(d^{-}(\alpha \cdot \beta) \cdot \gamma\right)=\int d^{-}(\alpha \cdot \beta) \cdot \gamma=\left\langle d^{-}(\alpha \cdot \beta), \gamma\right\rangle=0,
$$

\footnotetext{
${ }^{2}$ The definition of a Massey $l$-ple in a DGCA $\mathcal{A}^{*}$ is recalled in Definition $\mathrm{A} .2$
} 
by the orthogonality relation (2.5). As $\mathcal{A}^{*}$ is connected, $\int: \mathcal{H}^{7} \rightarrow \mathbb{R}$ is an isomorphism and so $m_{3}(\alpha, \beta, \gamma)=0$. The same argument shows that $m_{3}(\alpha, \beta, \gamma)=0$ if $\operatorname{deg}(\alpha, \beta, \gamma)=(4,2,2)$. The proof for the degree- 8 case is completely analogous.

The above corollary can be easily generalized to non-simply connected algebras of a simple kind.

Corollary 4.14. Let $\mathcal{A}^{*}$ be a connected Poincaré DGCA of degree n. Assume $\mathcal{A}^{*}=\mathcal{A}_{\mathrm{sc}}^{*}\left[t_{1}, \ldots, t_{k}\right]$, with $\mathcal{A}_{\mathrm{sc}}^{*}$ a simply connected Poincaré DGCA of degree $(n-k)$ of Hodge type, where $t_{1}, \ldots, t_{k}$ are variables in degree 1 with $d t_{i}=0$.

(1) If $n \leq 6$, then the Massey product of any Massey triple vanishes.

(2) If $n=7$, then the Massey product vanishes for every Massey triple of degree $\neq(2,2,2)$.

(3) If $n=8$, then the Massey product vanishes for every Massey triple of degree $\notin\{(2,2,2),(2,2,3),(2,3,2),(3,2,2)\}$.

Proof. For $k=0$ we are back to Corollary 4.13, so let us assume $k \geq 1$. If $n \leq$ 7 or if $n=8$ and $k \geq 2$, then $\mathcal{A}_{\mathrm{sc}}^{*}$ is formal by Corollary 3.11 , and so also $\mathcal{A}^{*}$ is formal. Namely, as $\mathcal{A}_{s c}^{*}$ is formal we have a zig-zag of quasi-isomorphisms of DGCAs $\mathcal{A}_{s c}^{*} \leftarrow \mathcal{D}_{1}^{*} \rightarrow \mathcal{D}_{2}^{*} \leftarrow \cdots \rightarrow H^{*}\left(\mathcal{A}_{s c}^{*}\right)$. As the variables $t_{i}$ are closed, this induces a zig-zag of quasi-isomorphisms of DGCAs $\mathcal{A}_{s c}^{*}\left[t_{1}, \ldots, t_{k}\right] \leftarrow$ $\mathcal{D}_{1}^{*}\left[t_{1}, \ldots, t_{k}\right] \rightarrow \mathcal{D}_{2}^{*}\left[t_{1}, \ldots, t_{k}\right] \leftarrow \cdots \rightarrow H^{*}\left(\mathcal{A}_{s c}^{*}\right)\left[t_{1}, \ldots, t_{k}\right]=H^{*}\left(\mathcal{A}_{s c}^{*}\left[t_{1}, \ldots, t_{k}\right]\right)$. So we are reduced to considering the case $\mathcal{A}^{*}=\mathcal{A}_{\mathrm{sc}}^{*}[t]$ with $\mathcal{A}_{\mathrm{sc}}^{*}$ a degree-7 simply connected Poincaré DGCA. As $d t=0$, we have $H^{*}\left(\mathcal{A}^{*}\right)=H^{*}\left(\mathcal{A}_{\mathrm{sc}}^{*}\right)[t]$ and the $A_{\infty}$-algebra structure on $H^{*}\left(\mathcal{A}^{*}\right)$ induced by the Kadeishvili-Merkulov construction is just the $\mathbb{F}[t]$-linear extension of the $A_{\infty}$-algebra structure on $H^{*}\left(\mathcal{A}_{\mathrm{sc}}^{*}\right)$. In particular, it is a $A_{3}$-algebra structure and

$$
\begin{aligned}
\mu_{3}([\alpha],[\beta],[\gamma])=\mu_{3}^{\mathrm{sc}} & {\left.\left[\alpha_{0}\right],\left[\beta_{0}\right],\left[\gamma_{0}\right]\right) } \\
& +(-1)^{\operatorname{deg}[\beta] \cdot[\gamma]} \mu_{3}^{\mathrm{sc}}\left(\left[\alpha_{-1}\right],\left[\beta_{0}\right],\left[\gamma_{0}\right]\right) t \\
& +(-1)^{\operatorname{deg}[\gamma]} \mu_{3}^{\mathrm{sc}}\left(\left[\alpha_{0}\right],\left[\beta_{-1}\right],\left[\gamma_{0}\right]\right) t \\
& +\mu_{3}^{\mathrm{sc}}\left(\left[\alpha_{0}\right],\left[\beta_{0}\right],\left[\gamma_{-1}\right]\right) t,
\end{aligned}
$$

where we have written $[x]=\left[x_{0}\right]+\left[x_{-1}\right] t$ for an homogeneous element $[x]$ in $H^{*}\left(\mathcal{A}^{*}\right)$. As $\operatorname{deg}\left[x_{0}\right]=\operatorname{deg}[x]$ and $\operatorname{deg}\left[x_{-1}\right]=\operatorname{deg}[x]-1$, by Corollary 4.13 we see that $\mu_{3}([\alpha],[\beta],[\gamma])$ will vanish unless $\operatorname{deg}([\alpha],[\beta],[\gamma]) \notin$ $\{(2,2,2),(2,2,3),(2,3,2),(3,2,2)\}$.

\section{Splitting OFF PARAllel 1-FOrmS}

In this section we generalize the Cheeger-Gromoll splitting theorem to the class of closed Riemannian manifolds whose harmonic 1-forms are parallel (Theorem 5.1, Remark 5.2). Using this result we extend results in the previous section that concern Massey triple products to the class of closed orientable Riemannian manifolds satisfying the condition of Theorem 5.1 
(Corollary [5.5) and derive the almost formality of compact $G_{2}$-manifolds, obtained by Chan-Karigiannis-Tsang in [CKT2018, from our results (Remark 5.6 .

Theorem 5.1. (Cheeger-Gromoll-type splitting) Let $\left(M^{n}, g\right)$ be a closed Riemannian manifold, and suppose that all harmonic 1-forms are parallel. Then there is a Riemannian fibration

$$
F^{n-k} \hookrightarrow M^{n} \longrightarrow T^{k},
$$

over a flat $k$-torus, where $k=b^{1}\left(M^{n}\right)$, and $F^{n-k}$ is closed. In fact, $M^{n}$ can be written as

$$
M^{n}=\left(\mathbb{R}^{k} \times F^{n-k}\right) / \Lambda,
$$

where $\Lambda \cong \mathbb{Z}^{k}$ acts isometrically on $F^{n-k}$ and by translations on $\mathbb{R}^{k}$. Moreover, $M^{n}$ is diffeomorphic (but not necessarily isometric) to a quotient of $\left(T^{k} \times F^{n-k}\right) / \Gamma$, where $\Gamma \subseteq \operatorname{Transl}\left(T^{k}\right) \times \operatorname{Diffeo}\left(F^{n-k}\right)$ is a finite abelian group.

Proof. Let $\varepsilon^{1}, \ldots, \varepsilon^{k}$ be the parallel 1-forms with dual vector fields $X_{i}:=$ $\left(\varepsilon^{i}\right)^{\#}$. Since the distribution spanned by the $\left(X_{i}\right)$ is parallel, so is its orthogonal complement, and the de Rham splitting theorem implies that the universal cover of $(M, g)$ is a Riemannian product

$$
\tilde{M}^{n}=\mathbb{R}^{k} \times \tilde{F}^{n-k},
$$

and the lifts $\tilde{X}_{i}$ of $X_{i}$ must be tangent to $\mathbb{R}^{k}$ and parallel, whence constant. As the deck transformations of the covering $\tilde{M}^{n} \rightarrow M$ must preserve $\tilde{X}_{i}$, they must act by translation on $\mathbb{R}^{k}$, whence the deck group $\Pi \cong \pi_{1}\left(M^{n}\right)$ must be contained in

$$
\Pi \subseteq \operatorname{Transl}_{\mathbb{R}^{k}} \times \operatorname{Isom}\left(\tilde{F}^{n-k}\right) .
$$

Let $\Pi_{0} \subseteq \Pi$ be the normal subgroup which acts trivially on $\mathbb{R}^{k}$, and let $F^{n-k}:=\tilde{F}^{n-k} / \Pi_{0}$. Then we may write

$$
\left(M^{n}, g\right)=\left(\mathbb{R}^{k} \times \tilde{F}^{n-k}\right) / \Pi=\left(\mathbb{R}^{k} \times F^{n-k}\right) / \Lambda,
$$

with a faithful map $\Lambda:=\Pi / \Pi_{0} \hookrightarrow \operatorname{Transl}_{\mathbb{R}^{k}}$, so that $\Lambda$ is abelian and torsion free, i.e., $\Lambda \cong \mathbb{Z}^{l}$ for some $l$. Therefore, $\Pi_{0}$ contains the commutator group $[\Pi, \Pi]$, so that there is a surjection $H_{1}\left(M^{n}\right)=\Pi /[\Pi, \Pi] \rightarrow \Lambda$, and as $H_{1}\left(M^{n}\right) / \operatorname{Tor}\left(H_{1}\left(M^{n}\right)\right) \cong \mathbb{Z}^{k}$ this means that $k \geq l$.

On the other hand, projection onto the first factor of $\mathbb{R}^{k} \times F^{n-k}$ implies that $\mathbb{R}^{k} / \Lambda$ is compact, whence $l=k$, and the image of the inclusion $\Lambda \hookrightarrow$ $\operatorname{Transl}_{\mathbb{R}^{k}} \cong \mathbb{R}^{k}$ must be a full lattice, so that $\mathbb{R}^{k} / \Lambda$ is a flat $k$-torus, and this projection yields the asserted fiber bundle structure.

Consider the homomorphism $\rho: \Lambda \cong \mathbb{Z}^{k} \rightarrow \operatorname{Isom}\left(F^{n-k}\right)$ from (5.1). The closure $G:=\overline{\rho(\Lambda)} \subseteq \operatorname{Isom}\left(F^{n-k}\right)$ is a compact abelian Lie group, as $\operatorname{Isom}\left(F^{n-k}\right)$ is a compact Lie group MS1939. In particular, $G$ has finitely many components. Thus, $\Lambda_{0}:=\rho^{-1}\left(G_{0}\right) \subseteq \Lambda$ is a subgroup of finite index, 
where $G_{0} \subseteq G$ denotes the identity component, and we have a Riemannian covering map

$$
\hat{M}^{n}:=\left(\mathbb{R}^{k} \times F^{n-k}\right) / \Lambda_{0} \longrightarrow M^{n}=\left(\mathbb{R}^{k} \times F^{n-k}\right) / \Lambda
$$

which is the quotient by a free action of the finite abelian group $\Gamma:=\Lambda / \Lambda_{0}$ on $\hat{M}^{n}$.

We finally have to show that $\hat{M}^{n}$ is diffeomorphic to $T^{k} \times F^{n-k}$. As $G_{0}$ is a compact connected abelian Lie group, it is isomorphic to the torus. Then $\left.\rho\right|_{\Lambda_{0}}: \Lambda_{0} \rightarrow G_{0}$ extends to a Lie group homomorphism $\bar{\rho}: \mathbb{R}^{k} \cong \Lambda_{0} \otimes \mathbb{R} \rightarrow$ $G_{0}$. Then

$$
T^{k} \times F^{n-k} \cong\left(\mathbb{R}^{k} / \Lambda_{0}\right) \times F^{n-k} \rightarrow\left(\mathbb{R}^{k} \times F\right) / \Lambda_{0}, \quad([v], f) \mapsto[(v, \bar{\rho}(v) f)]
$$

gives a diffeomorphism.

Remark 5.2. By Bochner's theorem [Bochner1946, all harmonic 1-forms on a Riemannian manifold with nonnegative Ricci curvature are parallel, so that Theorem 5.1 applies. In fact, the conclusion of Theorem 5.1 in the case of nonnegative Ricci curvature is also known as the Cheeger-Gromoll splitting theorem [CG1971, CG1972]. This holds, in particular, for $G_{2^{-}}$and Spin(7)-manifolds as these are Ricci flat.

Proposition 5.3. Let $\left(M^{n}, g\right)$ be as in Theorem 5.1, and orientable. Then $\Omega^{*}(M)$ is equivalent to $\left(\Lambda^{*} \mathcal{H}^{1}\left(M^{n}\right)\right) \otimes \mathcal{Q}^{*}=\mathcal{Q}^{*}\left[t_{1}, \ldots, t_{k}\right]$ with $k=b^{1}\left(M^{n}\right)$, where $\mathcal{Q}^{*}$ is a simply connected non-degenerate Poincaré DGCA of Hodge type of degree $(n-k)$.

Proof. By Theorem [5.1, $M^{n}$ is diffeomorphic to $\left(T^{k} \times F^{n-k}\right) / \Gamma$, where $\Gamma \subseteq$ $\operatorname{Transl}\left(T^{k}\right) \times \operatorname{Diffeo}\left(F^{n-k}\right)$ is a finite abelian group. Let $\Gamma^{\prime} \subseteq \operatorname{Diffeo}\left(F^{n-k}\right)$ be the projection of $\Gamma$ to the second factor, and choose a product metric $\tilde{g}:=g_{0} \oplus g_{1}$ on $T^{k} \times F^{n-k}$ such that $g_{0}$ is flat and $g_{1}$ is $\Gamma^{\prime}$-invariant. Then $\Gamma$ acts by isometries so that $\pi:\left(T^{k} \times F^{n-k}, \tilde{g}\right) \rightarrow\left(M^{n}, g\right)$ is a Riemannian covering for some metric $g$ on $M$. Clearly, the translation invariant fields on $T^{k}$ induce parallel vector fields on $M^{n}$, so that $\left(M^{n}, g\right)$ has $k=b^{1}\left(M^{n}\right)$ parallel vector fields. Thus, all harmonic 1-forms on $\left(M^{n}, g\right)$ are parallel.

As $\Gamma$ is a finite group, there is a projection map

$$
\pi_{\Gamma}: \Omega^{*}\left(T^{k} \times F^{n-k}\right) \longrightarrow \Omega^{*}\left(T^{k} \times F^{n-k}\right)^{\Gamma}, \quad \alpha \longmapsto \frac{1}{|\Gamma|} \sum_{\gamma \in \Gamma} \gamma^{*}(\alpha),
$$

and since $\Gamma$ acts by isometries, it follows that $\pi_{\Gamma}$ commutes with $d, d^{*}$ and $\triangle$. In particular, applying $\pi_{\Gamma}$ to the components of the Hodge decomposition of $\Omega^{*}\left(T^{k} \times F^{n-k}\right)$ induces a Hodge decomposition

$$
\begin{aligned}
\Omega^{*}\left(T^{k} \times F^{n-k}\right)^{\Gamma}= & d \Omega^{*-1}\left(T^{k} \times F^{n-k}\right)^{\Gamma} \oplus \mathcal{H}^{*}\left(T^{k} \times F^{n-k}\right)^{\Gamma} \\
& \oplus d^{*} \Omega^{*+1}\left(T^{k} \times F^{n-k}\right)^{\Gamma} .
\end{aligned}
$$

In the same way, we obtain the Hodge decomposition of $\mathcal{Q}^{*}:=\Omega\left(F^{n-k}\right)^{\Gamma^{\prime}}$

$$
\mathcal{Q}^{*}=d \mathcal{Q}^{*-1} \oplus \mathcal{H}^{*}\left(F^{n-k}\right)^{\Gamma^{\prime}} \oplus d^{*} \mathcal{Q}^{*+1} .
$$


Furthermore, since all $\alpha^{k} \in \Lambda^{*} \mathcal{H}^{1}\left(T^{k}\right)$ are parallel, we have for $\beta^{l} \in \mathcal{Q}^{*}$ : $*\left(\alpha^{k} \wedge \beta^{l}\right)= \pm\left(*_{T^{k}} \alpha^{k}\right) \wedge\left(*_{F^{n-k}} \beta^{l}\right) \in \mathcal{A}^{*}$, whence $d\left(\alpha^{k} \wedge \beta^{l}\right)= \pm \alpha^{k} \wedge d \beta^{l}$ and $d^{*}\left(\alpha^{k} \wedge \beta^{l}\right)= \pm \alpha^{k} \wedge d^{*} \beta^{l}$. Thus, (5.3) induces a Hodge decomposition of $\mathcal{A}^{*}:=\Lambda^{*} \mathcal{H}^{1}\left(T^{k}\right) \wedge \mathcal{Q}^{*} \cong \mathcal{Q}^{*}\left[t_{1}, \ldots, t_{k}\right]$

$$
\mathcal{A}^{*}=d \mathcal{A}^{*-1} \oplus\left(\Lambda^{*} \mathcal{H}^{1}\left(T^{k}\right) \wedge \mathcal{H}^{*}\left(F^{n-k}\right)^{\Gamma^{\prime}}\right) \oplus d^{*} \mathcal{A}^{*+1},
$$

and the $\Gamma$-invariant harmonic forms of the product metric on $T^{k} \times F^{n-k}$ decompose as

$$
\mathcal{H}^{*}\left(T^{k} \times F^{n-k}\right)^{\Gamma}=\left(\mathcal{H}^{*}\left(T^{k}\right) \wedge \mathcal{H}^{*}\left(F^{n-k}\right)\right)^{\Gamma}=\Lambda^{*} \mathcal{H}^{1}\left(T^{k}\right) \wedge \mathcal{H}^{*}\left(F^{n-k}\right)^{\Gamma^{\prime}},
$$

which thus coincides with the space of harmonic forms in $\mathcal{A}^{*}$ by (5.4). Therefore, the inclusion $\mathcal{A}^{*} \hookrightarrow \Omega^{*}\left(T^{k} \times F^{n-k}\right)^{\Gamma}$ is a quasi-isomorphism, and $\pi^{*}: \Omega^{*}\left(M^{n}\right) \rightarrow \Omega^{*}\left(T^{k} \times F^{n-k}\right)^{\Gamma}$ is an isomorphism, whence $\Omega^{*}\left(M^{n}\right)$ is quasi-isomorphic to $\mathcal{A}^{*}$ as claimed.

By the Künneth formula, $\operatorname{dim} H^{1}\left(\mathcal{A}^{*}\right)=k+\operatorname{dim} H^{1}\left(\mathcal{Q}^{*}\right)$, and as $\operatorname{dim} H^{1}\left(\mathcal{A}^{*}\right)=$ $b^{1}\left(M^{n}\right)=k$, it follows that $H^{1}\left(\mathcal{Q}^{*}\right)=0$, i.e., $\mathcal{Q}^{*}$ is a simply connected $(n-k)$-dimensional Poincaré algebra as asserted.

If $n-b^{1}(M) \leq 6$, then $\mathcal{Q}^{*}$ in Proposition 5.3 is formal by Corollary 3.11, whence so is $\mathcal{Q}^{*}\left[t_{1}, \ldots, t_{k}\right]$ by the same argument as in the proof of Corollary 4.14. Since this in turn is equivalent to $\Omega^{*}\left(M^{n}\right)$ by Proposition 5.3. we immediately obtain the following.

Corollary 5.4. Let $\left(M^{n}, g\right)$ be a closed orientable Riemannian manifold, and suppose that all harmonic 1-forms are parallel. If $n-b^{1}\left(M^{n}\right) \leq 6$, then $M^{n}$ is formal. In particular, any $G_{2}$-manifold with $b^{1}(M)>0$ (i.e., with holonomy strictly contained in $G_{2}$ ) and any Spin(7)-manifold with $b^{1}(M)>$ 1 is formal.

Corollary 5.5. Let $\left(M^{n}, g\right)$ be a closed oriented n-dimensional Riemannian manifold such that all harmonic 1-forms are parallel. Consider cohomology group $H^{*}(M, \mathbb{R})$.

(1) If $n \leq 6$, then the Massey product of any Massey triple vanishes.

(2) If $n=7$, then the Massey product vanishes for every Massey triple of degree $\neq(2,2,2)$.

(3) If $n=8$, then the Massey product vanishes for every Massey triple of degree $\notin\{(2,2,2),(2,2,3),(2,3,2),(3,2,2)\}$.

Proof. Assume that $\left(M^{n}, g\right)$ satisfies the condition of Corollary 5.5. By Proposition 5.3 the DGCA $\Omega^{*}(M)$ is equivalent to $\mathcal{Q}^{*}\left[t_{1}, \ldots, t_{k}\right], k=b^{1}(M)$, for a simply connected Poincaré DGCA $\mathcal{Q}^{*}$ of Hodge type. Then apply Corollary 4.14 .

Remark 5.6. Corollary 5.5 covers $G_{2^{-}}$and $\operatorname{Spin}(7)$-manifolds as these are Ricci-flat and hence have parallel harmonic 1-forms. Corollary 5.5 (2) for $G_{2}$-manifolds was first proved by [CKT2018, Theorem 4.15]. 


\section{Appendix A. Massey Products}

Let $\mathcal{A}^{*}$ be a DGCA and set $H^{*}=H^{*}\left(\mathcal{A}^{*}\right)$. For $x_{1}, \cdots, x_{l} \in H^{*}$, define the $l$ th order Massey product $\left\langle x_{1}, \cdots x_{l}\right\rangle$, which is a subset of $H^{\sum_{i=1}^{l}\left|x_{i}\right|+2-l}$, for $l \geq 3$ as follows.

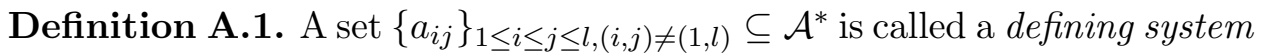
for the $l$ th order Massey product $\left\langle x_{1}, \cdots x_{l}\right\rangle$ if

- for $1 \leq i \leq l, a_{i i}$ represents $x_{i}$, and

- for $1 \leq i<j \leq l$ with $(i, j) \neq(1, l)$,

$$
d\left(a_{i j}\right)=\sum_{k=i}^{j-1} \bar{a}_{i k} a_{k+1, j},
$$

where $\bar{a}_{i k}=(-1)^{\left|a_{i k}\right|} a_{i k}$. Then we have the cocycle

$$
\alpha_{\left\{a_{i j}\right\}}=\sum_{k=1}^{l-1} \bar{a}_{1 k} a_{k+1, l} .
$$

The lth order Massey product $\left\langle x_{1}, \cdots x_{l}\right\rangle$ is defined by

$$
\left\langle x_{1}, \cdots x_{l}\right\rangle=\left\{\left[\alpha_{\left\{a_{i j}\right\}}\right] \mid\left\{a_{i j}\right\} \text { is a defining system }\right\} \subseteq H^{\sum_{i=1}^{l}\left|x_{i}\right|+2-l} .
$$

The Massey product $\left\langle x_{1}, \cdots, x_{l}\right\rangle$ is said to be trivial if it contains 0 .

Definition A.2. A Massey l-tuple (of $\mathcal{A}^{*}$ ) is an l-tuple $\left(x_{1}, \cdots, x_{l}\right)$ of cohomology classes such that $\left\langle x_{1}, \cdots, x_{l}\right\rangle \neq \emptyset$. We call $\left(\left|x_{1}\right|, \cdots,\left|x_{l}\right|\right)$ the degree of $\left(x_{1}, \cdots, x_{l}\right)$.

Lemma A.3. Let $\left(x_{1}, \cdots, x_{l}\right)$ be a Massey l-tuple for $l \geq 3$. Then the lth order Massey product $\left\langle x_{1}, \cdots, x_{l}\right\rangle$ is trivial if one of the following holds.

(1) We have $x_{p}=0$ for some $p$.

(2) A DGCA $\mathcal{A}^{*}$ is connected and $\left|x_{p}\right|=0$ for some $p$.

(3) ADGCA $\mathcal{A}^{*}$ is a connected Poincaré DGCA of degree $n$ and $\sum_{i=1}^{l}\left|x_{i}\right|+$ $2-l=n$.

Proof. By[Kraines1966, (2.3)], we have $c\left\langle x_{1}, \cdots, x_{l}\right\rangle \subset\left\langle x_{1}, \cdots, c x_{p}, \cdots, x_{l}\right\rangle$ for any $c \in \mathbb{F}$. Then (1) is immediate.

Next, we show (2). As $\mathcal{A}^{*}$ is connected, the product in cohomology $H^{0}\left(\mathcal{A}^{*}\right) \otimes H^{k}\left(\mathcal{A}^{*}\right) \rightarrow H^{k}\left(\mathcal{A}^{*}\right)$ is the multiplication by scalars on $H^{k}\left(\mathcal{A}^{*}\right)$ by Remark 2.7. We also have $x_{p-1} \cdot x_{p}=x_{p} \cdot x_{p+1}=0$ by (A.1). Then we see that $x_{p}=0$ or $x_{p-1}=x_{p+1}=0$. Hence (2) follows from (1).

Finally, we prove (3). Fix a defining system $\left\{a_{i j}\right\}$. As $a_{2 l}$ does not appear in the right hand side of (A.1),$\left\{a_{i j}+\delta_{i 2} \delta_{j l} \gamma\right\}$ for any cocycle $\gamma \in \mathcal{A}^{\left|a_{2 l}\right|}$ is also a defining system. As $\left[a_{11}\right]=x_{1}$, we see that

$$
\left\langle x_{1}, \cdots, x_{l}\right\rangle \supset \alpha_{\left\{a_{i j}\right\}}+x_{1} \cdot H^{\sum_{i=2}^{l}\left|x_{i}\right|+2-l}=\alpha_{\left\{a_{i j}\right\}}+x_{1} \cdot H^{n-\left|x_{1}\right|} .
$$

When $x_{1}=0,\left\langle x_{1}, \cdots, x_{l}\right\rangle$ is trivial by (1). When $x_{1} \neq 0$, we have $x_{1}$. $H^{n-\left|x_{1}\right|} \neq 0$ by the non-degeneracy of $\langle-,-\rangle$ defined in Definition 2.1 (1). 
This implies that $x_{1} \cdot H^{n-\left|x_{1}\right|}=H^{n}$ as $\operatorname{dim} H^{n}=1$, and hence $\left\langle x_{1}, \cdots, x_{l}\right\rangle=$ $H^{n} \ni 0$.

Proposition A.4. Let $\mathcal{A}^{*}$ be a $(r-1)$-connected $(r>1)$ Poincaré DGCA of degree $n$. For $l \geq 3$, the following holds.

(1) If $n \leq r(l+1)+(1-l)$, the $l^{\prime}$ th order Massey product of any Massey $l^{\prime}$-tuple is trivial for any $l^{\prime} \geq l$.

(2) If $n=r(l+1)+(2-l)+q$ for $q \geq 0$, then the lth order Massey product is trivial for every Massey l-tuple of degree $\notin\left\{\left(r+a_{1}, \cdots, r+a_{l}\right) \mid\right.$ $\left.a_{i} \geq 0,0 \leq \sum_{i=1}^{l} a_{i} \leq q\right\}$.

Proof. Let $\left(x_{1}, \cdots x_{l}\right)$ be a Massey l-tuple. By Lemma A.3, we must have

$$
\left|x_{i}\right| \geq r \quad \text { for any } i, \quad \text { and } \quad \sum_{i=1}^{l}\left|x_{i}\right|+2-l \leq n-r
$$

so that $\left\langle x_{1}, \cdots, x_{l}\right\rangle$ is nontrivial because $H^{k}=0$ for $1 \leq k \leq r-1, n-r+1 \leq$ $k \leq n-1$ and $k \geq n+1$. In particular, we must have

$$
r l+2-l \leq n-r \quad \Longleftrightarrow \quad n \geq r(l+1)+(2-l),
$$

which proves (1). Next, we prove (2). By the first equation of (A.2), we must have $\left|x_{i}\right|=r+a_{i}$ for $a_{i} \geq 0$. Then the second equation becomes

$$
r l+\sum_{i=1}^{l} a_{i}+2-l \leq r l+(2-l)+q \quad \Longleftrightarrow \quad 0 \leq \sum_{i=1}^{l} a_{i} \leq q,
$$

which proves (2).

The following is immediate from Proposition A.4.

Corollary A.5. Let $\mathcal{A}^{*}$ be a $(r-1)$-connected $(r>1)$ Poincaré DGCA of degree $n$. If $n \leq 4 r-2$, all of the Massey products are trivial. If $n \leq 5 r-3$, all of the $l$ th order Massey products are trivial for $l \geq 4$.

Note that we obtain Corollary 4.13 also from Proposition A.4.

Acknowledgement. HVL and LS thank the VIASM in Hanoi and MPIMIS in Leipzig for hospitality and excellent working conditions during their visit in 2018, where a part of this project was discussed. KK thanks Hisashi Kasuya for explaining Sullivan's work. The authors wish to thank Diarmuid Crowley and Johannes Nordström for discussions on [CN2015] and Pavel Hajek for helpful comments on the relation of this article to Hajek2018, [LS2007, Theorem 1.1] and [CFL2015, Lemma 11.1]. 


\section{REFERENCES}

[Barr1968] M. BARR, Harrison homology, Hochschild homology and triples, J. Algebra, 8 (1968), 314-323.

[Bochner1946] S. Bochner, Vector fields and Ricci curvature, Bull. Amer. Math. Soc., 52 (1946), 776-797.

[BMFM2018] U. BuiJs, J. M. Moreno-Fernández, And A. Murillo, A-infinity structures and Massey products, arXiv:1801.03408.

[Cavalcanti2006] G. Cavalcanti, Formality of $k$-connected spaces in $4 k+3$ - and $4 k+4$ dimensions, Math. Proc. Camb. Phil. Soc., 141 (2006), 101-112.

[CFL2015] K. Cieliebak, K. Fukaya and J. Latschev, Homological algebra related to surfaces with boundary, arXiv:1508.02741.

[CG1971] J. Cheeger And D. Gromoll, The splitting theorem for manifolds of nonnegative Ricci curvature, J. of Diff. Geometry, 6 (1971), 119-128.

[CG1972] J. Cheeger and D. Gromoll, On the structure of complete manifolds of nonnegative curvature, Annals of Math., 96 (1972), 413-433.

[CKT2018] K.F. Chan, S. Karigiannis and C.C. Tsang, The $\mathcal{L}_{B}$-cohomology on compact torsion-free $\mathrm{G}_{2}$ manifolds and an application to 'almost' formality, arXiv:1801.06410, to appear in Ann. Global Anal. Geom.

[CN2015] D. Crowley, J. Nordström, The rational homotopy type of $(n-1)$-connected manifolds of dimension up to $5 n-3$, arXiv:1505.04184 2 .

[DGmS1975] P. Deligne, P. Griffiths, J. Morgan and D. Sullivan, Real homotopy theory of Kähler manifolds, Invent. Math., 29 (1975), 245-274.

[Hajek2018] P. HAJEK, Twisted IBL $_{\infty}$-algebra and string topology: First look and examples, arXiv:1811.0528.

[Harrison1962] D. K. HARRISOn, Commutative algebras and cohomology. Trans. Amer. Math. Soc., 104 (1962), 191-204.

[Kadeishvili1980] T. V. KADEISHVILI, On the theory of homology of fiber spaces, Uspekhi Mat. Nauk, 35 (1980), 183-188, International Topology Conference (Moscow State Univ., Moscow, 1979).

[Kadeishvili1988] T. V. Kadeishvili, Structure of $A(\infty)$-algebra and Hochschild and Harrison cohomology, Proc. of A. Razmadze Math. Inst., 91 (1988), 20-27.

[Kadeishvili2009] T. V. KADEIShvili, Cohomology $C_{\infty}$-algebra and rational homotopy type, Algebraic topology, old and new, Banach Center Publ., vol. 85, Polish Acad. Sci. Inst. Math., Warsaw, (2009), 225-240.

[Kaledin2005] D.KALEDIN, Some remarks on formality in families, arXiv:math/0509699.

[Kontsevich1994] M. Kontsevich, Feynman diagram and low dimensional topology, Proc. of the first European Congr. Math. I, (1994), 97-121.

[KS2001] M. Kontsevich, Y. Soibelman, Homological mirror symmetry and torus fibrations, Symplectic geometry and mirror symmetry (Seoul, 2000), 203-263, World Sci. Publ., River Edge, NJ, 2001.

[Kotschick2001] D. Kotschick, On products of harmonic forms, Duke Math. J., 107 (2001), 521-531.

[Kraines1966] D. Kraines, Massey higher products, Trans. Amer. Math. Soc., 124 (1966), 431-449.

[LS2007] P. Lambrechts And D. Stanley, Poincaré duality and commutative differential graded algebras, Annales Scientifiques de l'École Nomale Supérieure, quatrième série - tome 41 fascicule 4 juillet-août 2008.

[Loday1992] Loday, Jean-Louis. Cyclic homology. Grundlehren der Mathematischen Wissenschaften, 301. Springer-Verlag, Berlin, 1992.

[LV2012] J.-L. LodAy And B. VAllette, Algebraic operads, Grundlehren der Mathematischen Wissenschaften, 346, Springer-Heidelberg, 2012.

[Lunts2007] V. A. Lunts, Formality of DG algebras (after Kaledin), arXiv:0712.0996 
[Merkulov1999] S.A. Merkulov, Strong homotopy algebras of a Kähler manifold, Internat. Math. Res. Notices, (1999), 153-164.

[MS1939] Myers AND SteEnrod, The group of isometries of a Riemannian manifold, Ann. of Math. (2), 40 (1939), 400-416.

[Miller1979] T. J. Miller, On the formality of $k-1$ connected compact manifolds of dimension less than or equal to $4 k-2$, Illinois J. Math., 23 (1979), 253-258.

[Sullivan1975] D. Sullivan, Differential forms and the topology of manifolds, ManifoldsTokyo (1973) (Proc. of the Intern. Conf. on Manifolds and related topics in Topology, Tokyo 1973) (ed. A. Hattori), U. of Tokyo Press, 1975, 37-49.

[Sullivan1977] D. Sullivan, Infinitesimal computations in topology. Publ. Math. IHES, 47 (1977), 269-331.

[Valette2012] B. VAllette, Algebra+Homotopy=Operad, arXiv:1202.3245.

Dipartimento di Matematica "Guido Castelnuovo", Università di Roma "La Sapienza", Piazzale Aldo Moro 2, 00185 Roma, Italy

Email address: fiorenza@mat.uniroma1.it

Gakushuin University, 1-5-1, Mejiro, Toshima, Tokyo, 171-8588, Japan

Email address: kkawai@math.gakushuin.ac.jp

Institute of Mathematics, Czech Academy of Science, Zitna 25, 11567 Praha

1, Czech Republic

Email address: hvle@math.cas.cz

Faculty for Mathematik, TU Dortmund University, Vogelpothsweg 87, 44221 Dortmund, Germany

Email address: 1schwach@math.tu-dortmund.de 